\title{
Lagrangian studies of wave-induced flows in a viscous ocean
}

\author{
Jan Erik H. Weber ${ }^{1}$ \\ ${ }^{1}$ Department of Geosciences, University of Oslo, PO Box 1022, Blindern, NO-0315 Oslo, \\ Norway
}

ABSTRACT

A review of the Lagrangian approach to wave-induced drift in a rotating fluid layer of finite depth is presented. The Lagrangian description of fluid motion is (usually) more mathematically demanding than the traditional Eulerian approach. However, it yields directly the mean particle drift velocity in periodic motion. The solution to the wave-drift problem in a Lagrangian description depends crucially on the viscosity $v$ being non-zero, however small. It illustrates the singular nature of this problem, in which the limit of solutions as $v \rightarrow 0$ is different from solutions obtained with $v=0$. Obviously, for most oceanographic applications, the effect of an (eddy) viscosity cannot be neglected. In this survey we consider solutions for the Lagrangian mean drift in various types of surface waves influenced by viscosity. From a purely Lagrangian starting point, we demonstrate novel ways of deriving conservation equations for the mean wave momentum and the mean wave energy in a weakly viscous fluid layer of finite depth. Among several examples of mean drift in surface waves, we consider deep water gravity waves acted upon by an oscillating wind stress, short capillary-gravity waves affected by a thin elastic surface film, and friction-induced roll

\footnotetext{
${ }^{1}$ Corresponding author. Tel.: 47 22855826; fax: 4722855269.

E-mail address: j.e.weber@geo.uio.no (J.E. Weber)
} 
motion in short-crested gravity waves. Furthermore, the drift in high-frequency shallow-water gravity waves is revisited, and a new equation for the Lagrangian mean drift in temporally/spatially modulated waves is presented. Finally, we discuss the use of the CoriolisStokes force to implement Lagrangian properties into Eulerian numerical ocean circulation models.

\section{Introduction}

The idea of attempting to determine the history of every particle in a fluid was originally due to Euler (1757). He introduced the form of the hydrokinetic equations that later should become known as the Lagrangian form (as well as the alternative and more used Eulerian notation). The fact that the Lagrangian approach has been considered more cumbersome than the Eulerian formulation, may explain why relatively few studies of ocean flows based on the Lagrangian formalism are found in the early literature. Among these few studies the exact solution by Gerstner (1809) for rotational surface waves in an infinitely deep ocean stands out. Later Stokes (1847) considered flow between material surfaces in irrotational surface waves to obtain the wave-induced mean drift (the Stokes drift), but he did not apply a direct Lagrangian formulation. Much later Miche (1944) used the Lagrangian form in his study of irrotational and rotational surface waves in a fluid layer of finite depth.

Post-war studies are more numerous, as will be shown below. In particular we mention that recent progress in Lagrangian tracking of balloons in the atmosphere and floats in the ocean has spurred the theoretical development of Lagrangian dispersion theory; see the comprehensive account by Bennet (2006). A very recent review of Lagrangian analysis used to study the output of ocean circulation models and ocean velocity data from altimetry is 
found in Sebille et al. (2018).

The present survey is devoted to a Lagrangian description of ocean dynamics, and in particular how periodic waves induce mean flows within a purely Lagrangian framework. In this connection it is worth mentioning that the theory of nonlinear waves on a Lagrangianmean flow (GLM theory) by Andrews and McIntyre (1978) is a hybrid Lagrangian formulation. The averaging operator used to arrive at the Lagrangian-mean variables in the GLM theory is not a full particle-following Lagrangian mean, but an Eulerian mean of the variables evaluated at their displaced locations. Although useful and elegant, the GLM theory will not be discussed further in this survey. Again, those who are mainly interested in turbulent dispersion are referred to Bennet's book.

In this review we focus on the Lagrangian drift in high-frequency ocean waves, i.e. waves with frequency much higher than the inertial frequency. That means that drift in lowfrequency shallow-water waves of the tidal type (Poincaré waves); see e.g. Høydalsvik and Weber (2003), will not be discusses here.

\subsection{Inviscid flow}

In addition to the already mentioned papers by Gerstner (1809) and Miche (1944), a considerable amount of studies on surface waves in Lagrangian form have been published under the assumption that the fluid is inviscid; see e.g. Kravtchenko and Daubert (1957), Abrashkin (1996), Constantin (2001, 2012, 2014, 2015), Clamond (2007), Chen et al. (2010), Weber (2012), Henry (2013, 2016), Ionescu-Kruse (2015), and Abrashkin and Pelinovsky (2017). The basic diversion in this case is between waves with vorticity of the Gerstner-type, and irrotational waves of the Stokes-type. In the first case the particle orbits are closed, i.e. no mean drift, while in the second case there is a Stokes drift in the wave propagation direction. 
However, in a rotating ocean the action of the Coriolis force makes the particles associated with the mean motion in the Stokes wave to move in closed inertial circles. Hence, when averaged over the inertial period, there is no net forward drift in such waves (Ursell, 1950; Hasselmann, 1970). This is also the fact for inviscid surface waves with vorticity (Pollard, 1970; Constantin and Monismith, 2017).

\subsection{The effect of viscosity}

The presence of viscosity has a profound effect on the wave-induced drift. In a pioneering paper (Longuet-Higgins, 1953) it was demonstrated that the inclusion of a small viscosity not only modified the motion in thin boundary layers near the surface and at the bottom, but also produced significant changes from Stokes' solution in the interior. Most notably, the mass transport velocity gradient just below the surface boundary layer is found to be twice the value obtained from Stokes' irrotational solution. Furthermore, above the viscous bottom boundary layer the mean drift velocity turns out to be $5 / 2$ times the inviscid Stokes drift.

It was Pierson (1962) that really opened up for the use of a Lagrangian description in viscous ocean flows, introducing a perturbation expansion of the Lagrangian equations. Although he just discussed some first order solutions, nonlinear Lagrangian analyses, particularly of surface waves, soon followed (Chang, 1969; Ünlüata and Mei, 1970; Madsen, 1978; Weber, 1983a,b; Jenkins, 1986, 1987). For Lagrangian approaches to two horizontal fluid layers of different densities, the effect of viscosity on the drift in interfacial waves has been considered by Weber and Førland (1990) for short temporally and spatially attenuated waves in an air-water system. Later Piedra-Cueva (1995) studied the steady two-layer problem in the Lagrangian formulation with emphasis on transport in a very viscous (mud) bottom layer induced by spatially damped surface water waves. A similar problem for the 
steady drift in partial standing surface waves in a two-layer viscous system was investigated by Ng (2004a). Moreover, Ng and Zhang (2007) used a direct Lagrangian approach for studying the mass transport in progressive water waves over a layer of viscoelastic mud.

In the upper ocean the currents are predominantly driven by the wind. But some of the atmospheric momentum is transferred to surface waves. We will not go into the various theories of how surface waves are generated by the wind. The pioneering studies here are Miles (1957) and Phillips (1957). In this survey we consider fully developed surface waves, and take that the waves are monochromatic. However, it is fairly simple to generalize, and express the wave-forcing in a real sea state by its spectral form (Jenkins, 1989; McWilliams and Restrepo, 1999; Weber et al., 2006).

For monochromatic waves it can be shown that if the wind stress contains an undulating part that performs work on the waves, the loss of wave energy due to viscous dissipation may be exactly compensated (Lamb, 1932). In this case the waves propagate with constant amplitude, i.e. they are so-called permanent waves. On the other hand, the wind may also promote wave growth, which finally leads to breaking, or it may cause enhanced damping. The damping is particularly pronounced for adverse winds. The wave-induced drift in such cases has been investigated by Weber and Melsom (1993). In the case of breaking, the transition of accumulated mean wave momentum to ocean currents has been studied among others by Melsom (1996) and Melsom and Saetra (2004).

The mean wind stress at the ocean surface causes mean momentum to diffuse into the ocean to a certain depth till it finally is balanced by the Coriolis force due to the earth's rotation, as first shown by Ekman (1905). This balance occurs within the so-called Ekman layer. But the presence of wind inevitably leads to surface waves. When wind-generated surface waves propagate out of the storm area and into regions not influenced by wind, they will attenuate in time or space due to friction. Hence, some of the initial mean wave 
momentum in irrotational surface waves (the Stokes flux) will reappear as Eulerian mean currents. This occurs through the action of the virtual wave stress, first defined by LonguetHiggins (1969). Therefore, in a viscous ocean, the Lagrangian mean mass transport due to waves must be written as a sum of the Stokes drift and a frictionally-induced Eulerian mean current (Longuet-Higgins, 1953).

For surface waves in a viscous ocean, the viscosity is small, and the effect is confined to a thin viscous boundary layer (millimeters to centimeters) just beneath the wavy surface. A Cartesian Eulerian approach in this case would actually require that the wave amplitude is smaller than the boundary-layer thicknesses, which limits the wave amplitude quite severely; see e.g. Craik (1982). To remedy this one could use curvilinear coordinates, following the wavy interface, but this is problematic since the wave amplitude in our problem grows/attenuates in space and time, depending on the atmospheric conditions. A better option is to use our particle-following Lagrangian coordinates. Then the surface wave amplitude can be much larger than the boundary layer thickness, but small enough for a series expansion in the wave steepness to be valid. It should also be mentioned that hybrid methods, with a Lagrangian coordinate system in the vertical and an Eulerian coordinate system in the horizontal has been introduced to present a simpler formulation than the three dimensional Lagrangian mean equations (see e.g. Mellor, 2003, 2005; Broström et al., 2008; Aiki and Greatbatch , 2012).

\section{Mathematical formulation}

We consider motion in an incompressible viscous fluid layer of constant depth $H$. The motion is described by using a Lagrangian formulation. Let a fluid particle $(a, b, c)$ initially have coordinates $\left(X_{0}, Y_{0}, Z_{0}\right)$. Its position $(X, Y, Z)$ at later times will then be a function of 
$a, b, c$ and time $t$. Here the axes of $X$ and $Y$ are horizontal, and situated at the undisturbed surface, while the vertical $Z$-axis is positive upwards. Velocity components and accelerations are given by $\left(X_{t}, Y_{t}, Z_{t}\right)$ and $\left(X_{t t}, Y_{t t}, Z_{t t}\right)$, respectively, where subscripts denote partial differentiation. The initial density of a particle is $\rho_{0}$, and the density at subsequent times is $\rho$. We follow the traditional approach by representing the rotation of the ocean by its vertical angular velocity $f / 2$, where $f$ is the Coriolis parameter; see Gerkema et al. (2008) for a thorough discussion of the implementation of the Coriolis force in a rotating ocean.

Furthermore, in this survey we take that $f$ is constant, so problems that requires the $\beta$-plane approximation, are not considered here.

\subsection{Conservation of mass}

The conservation of mass in Lagrangian form becomes; see e.g. Lamb (1932):

$\rho \partial(X, Y, Z) / \partial(a, b, c)=\rho_{0} \partial\left(X_{0}, Y_{0}, Z_{0}\right) / \partial(a, b, c)$

where $\partial() / \partial(a, b, c)$ is the Jacobian operator. For an incompressible fluid, the density is conserved for a particle, i.e. $\rho=\rho_{0}$ for all times, and hence

$$
\partial(X, Y, Z) / \partial(a, b, c)=\partial\left(X_{0}, Y_{0}, Z_{0}\right) / \partial(a, b, c) .
$$

This expresses the conservation of volume for a fluid particle, and is often referred to as the continuity equation. In this survey we take that the fluid is incompressible and homogenous, so the density is constant. By defining

$$
D_{0} \equiv \partial\left(X_{0}, Y_{0}, Z_{0}\right) / \partial(a, b, c)
$$


we note that further simplifications can be introduced by assuming $D_{0}=1$. Lagrangian variables that fulfill this condition are sometimes called Miche's coordinates (Clamond, 2007). However, this simplification should be done with care. For example, in the famous exact Gerstner solution for rotational waves (Gerstner, 1809), we have $D_{0} \neq 1$.

\subsection{Conservation of momentum}

The viscous version of the Lagrangian equations in a non-rotating system is found in Pierson (1962). Here the fluid viscosity is assumed to be constant. Introducing the Coriolis force in Pierson (1962), and also assuming that generally $D_{0} \neq 1$ in (3), the equation for the conservation of momentum can be written:

$$
\begin{aligned}
& X_{t t}-f Y_{t}=-D_{0}^{-1} \partial(P / \rho, Y, Z) / \partial(a, b, c)+v \nabla^{2} X_{t} \\
& Y_{t t}+f X_{t}=-D_{0}^{-1} \partial(X, P / \rho, Z) / \partial(a, b, c)+v \nabla^{2} Y_{t} \\
& Z_{t t}+g=-D_{0}^{-1} \partial(X, Y, P / \rho) / \partial(a, b, c)+v \nabla^{2} Z_{t}
\end{aligned}
$$

where $P$ is the pressure, $g$ is the acceleration due to gravity, and $v$ is the constant kinematic eddy viscosity. The explicit Lagrangian form of the Laplacian operator $\nabla^{2}$ in (4)-(6) can be found in Pierson (1962).

For oceanic applications, the concept of a constant eddy viscosity is somewhat problematic since diffusion of momentum is related to turbulent mixing (apart from very thin molecular sub-layers). In the surface layer and near the ocean bottom this mixing varies considerably in space, particularly in the vertical direction. This problem is not specific for the Lagrangian description. It is exactly the same for the more common Eulerian way of modelling oceanic (and atmospheric) flows. However, since the spatial variations in the 
Lagrangian description are nonlinear, the mathematical difficulties related to higher order solutions with a variable turbulent (eddy) viscosity soon becomes rather severe. There are a few attempts to apply a variable eddy viscosity to wave-induced drift problems; see e.g. Jenkins (1987, 1989), Weber and Melsom (1993), Melsom (1993). The general effect is to produce surface drift currents that are somewhat less deflected from the wind and wave direction than those obtained for a constant eddy viscosity. We shall, in specific examples, modify (4)-(6) to encompass a vertically-varying eddy viscosity.

\subsection{Method of solution}

Due to the strong nonlinear character of the governing equation in Lagrangian form, exact analytical solutions are generally not obtainable. In such cases one can try to write the solutions as series expansions after a small parameter $\varepsilon$ (Pierson, 1962). Without the need to specify $\varepsilon$, we may formally write

$$
\left.\begin{array}{l}
X=a+\varepsilon x^{(1)}+\varepsilon^{2} x^{(2)}+\varepsilon^{3} x^{(3)}+\cdots \\
Y=b+\varepsilon y^{(1)}+\varepsilon^{2} y^{(2)}+\varepsilon^{3} y^{(3)}+\cdots \\
Z=c+\varepsilon z^{(1)}+\varepsilon^{2} z^{(2)}+\varepsilon^{3} z^{(3)}+\cdots \\
P=P_{0}-\rho g c+\varepsilon p^{(1)}+\varepsilon^{2} p^{(2)}+\varepsilon^{3} p^{(3)}+\cdots
\end{array}\right\}
$$

where $P_{0}$ is a constant. In wave problems the small parameter will usually be proportional to the wave steepness, and accordingly, the solutions obtained from (7) will apply to smallamplitude waves. However, surface waves in the open ocean are not very steep. As far as wind waves are concerned, the amplitudes are kept small due to repeated breaking. In that case $O\left(\varepsilon^{2}\right)$ theory yields results for the drift currents in (basically) irrotational waves that compares in magnitude with the purely wind-driven Ekman current (Weber, 1983b; Weber and Melsom, 1993; Ardhuin et al., 2009; Röhrs et al., 2012). 
We generally consider plane waves that propagate along the $X$-axis. However, in Section 8 we extend the discussion to a pair of obliquely propagating waves, yielding a short-crested surface wave pattern. Then the solution will display a dependence of both horizontal coordinates $a$ and $b$. For the remaining part of the paper, we consider waves with infinitely long crests in the $Y$-direction. In that case it is convenient to introduce the notation

$$
\left.\begin{array}{l}
X=a+x(a, c, t), \\
Y=b+y(a, c, t), \\
Z=c+z(a, c, t), \\
P=P_{0}-\rho g c+p(a, c, t),
\end{array}\right\}
$$

where the deviations $x, y, z, p$, representing the series expansion parts of (7), do not depend on the coordinate $b$.

The conservation of volume (2) now reduces to:

$x_{a}+z_{c}+J(x, z)=x_{0 a}+z_{0 c}+J\left(x_{0}, z_{0}\right)$,

where $J$ is the two-dimensional Jacobian given by $J(A, B) \equiv A_{a} B_{c}-A_{c} B_{a}$. Subscript zero here denotes initial deviations. To $O(\varepsilon)$ we have from (9) that $x_{t a}+z_{t c}=0$, and hence $x_{a}+z_{c}=0$ for a linear progressive wave. It then follows that $x_{0 a}+z_{0 c}=0$ to this order. Accordingly, in (3):

$D_{0}=1+O\left(\varepsilon^{2}\right)$.

The momentum equations (4)-(6), correct to $O\left(\varepsilon^{2}\right)$, then become

$$
\begin{aligned}
x_{t t}-f y_{t}= & -(p / \rho+g z)_{a}-J(p / \rho, z)+v \nabla_{L}^{2} x_{t} \\
& -v\left(2 x_{a} x_{t a a}+2 z_{c} x_{t c c}+2\left(z_{a}+x_{c}\right) x_{t a c}\right. \\
& \left.+x_{t a} \nabla_{L}^{2} x+x_{t c} \nabla_{L}^{2} z\right),
\end{aligned}
$$

$y_{t t}+f x_{t}=v \nabla_{L}^{2} y_{t}$ 


$$
\begin{aligned}
z_{t t}= & -(p / \rho+g z)_{c}-J(x, p / \rho)-g J(x, z)+v \nabla_{L}^{2} z_{t} \\
& -v\left(2 x_{a} z_{t a a}+2 z_{c} z_{t c c}+2\left(z_{a}+x_{c}\right) z_{t a c}+z_{t a} \nabla_{L}^{2} x+z_{t c} \nabla_{L}^{2} z\right) .
\end{aligned}
$$

Here $\nabla_{L}^{2}=\partial^{2} / \partial a^{2}+\partial^{2} / \partial c^{2}$ is the linear part of the Laplacian operator in Lagrangian form.

The periodic wave solutions of (11)-(13) to $O(\varepsilon)$ will be denoted by a tilde, while second order mean quantities, where the mean is defined by the average over the wave cycle, will be denoted by an overbar.

\subsection{Boundary conditions}

In the absence of waves, it is often convenient to label the ocean surface in Lagrangian notation by $c=0$. But this is a material surface, i.e. it always consists of the same particles unless it is disrupted by mixing processes. Therefore, in the presence of non-breaking waves, whatever amplitude, the free surface is still given by $c=0$. This is a considerable advantage compared to the nonlinear kinematic boundary condition at the free surface in an Eulerian description, relating the vertical velocity to the material rate of change of the Eulerian surface position.

The tangential and normal atmospheric stresses at the surface $c=0$ are given by $\left(\tau_{1}, \tau_{2}, \sigma\right)$, respectively. We assume that our primary wave has a real frequency $\omega$ that is much larger than the inertial frequency $f$. Then we can disregard the effect of the earth's rotation on the primary wave field, which means that we have no velocity along the $Y$-axis to this order. Using that $\tilde{y}=0$, and $\partial / \partial b=0$ for waves along the $X$-axis, the dynamic surface boundary conditions for the periodic motion to $O(\varepsilon)$ become (neglecting the effect of surface films for the moment): 


$$
\left.\begin{array}{l}
\tilde{\tau}_{1}=\rho v\left(\tilde{x}_{t c}+\tilde{z}_{t a}\right) \\
\tilde{\tau}_{2}=0 \\
\tilde{\sigma}=-\tilde{p}+2 \rho v \tilde{z}_{t c}
\end{array}\right\}, c=0 .
$$

The dynamic boundary conditions for the mean motion to $O\left(\varepsilon^{2}\right)$, allowing for a drift in the $Y$ direction due to the Coriolis force, can be written

$$
\left.\begin{array}{l}
\bar{\tau}_{1}=\rho v\left(\bar{x}_{t c}+\bar{z}_{t a}+\overline{\tilde{x}_{t c} \tilde{x}_{a}}-\overline{\tilde{x}_{t a} \tilde{x}_{c}}+\overline{\tilde{z}_{t a} \tilde{z}_{c}}-3 \overline{\tilde{x}_{t a} \tilde{z}_{a}}\right) \\
\bar{\tau}_{2}=\rho v \bar{y}_{t c} \\
\bar{\sigma}=-\bar{p}+2 \rho v\left(\bar{z}_{t c}+\overline{\tilde{z}_{t c} \tilde{x}_{a}}-\overline{\tilde{z}_{t a} \tilde{x}_{c}}-\overline{\tilde{x}_{t c} \tilde{z}_{a}}-\overline{\tilde{z}_{t a} \tilde{z}_{a}}\right)
\end{array}\right\}, c=0 .
$$

Here we have used that $\tilde{x}_{a}=-\tilde{z}_{c}$. At the bottom, given by by $c=-H$, we apply a no-slip condition for both the periodic and the mean motion:

$\left.\begin{array}{l}\tilde{x}_{t}=\tilde{z}_{t}=0 \\ \bar{x}_{t}=\bar{y}_{t}=\bar{z}_{t}=0\end{array}\right\}, \quad c=-H$.

Large parts of the ocean surface may at times be covered by thin elastic films of biogenic or anthropogenic origin. Such films will strongly affect short capillary-gravity waves. Mathematically, the effects are manifested through additional terms in the surface boundary conditions. We return to this problem in Section 7.

\section{The primary field for high frequency waves}

We here consider ocean surface waves with frequencies that are much higher than the inertial frequency. Hence the linear wave field is not influenced by the earth's rotation. In general, for a viscous fluid, we may write the linear horizontal and vertical wave velocity as a sum of a potential part $\tilde{\varphi}$ and a vorticity part $\tilde{\psi}(\mathrm{Lamb}, 1932)$ :

$$
\tilde{x}_{t}=-\tilde{\varphi}_{a}-\tilde{\psi}_{c}, \tilde{z}_{t}=-\tilde{\varphi}_{c}+\tilde{\psi}_{a}
$$


Substituting from (17) into the linearized versions of (11) and (13) we obtain

$$
\left.\begin{array}{l}
\nabla_{L}^{2} \tilde{\varphi}=0, \\
\tilde{\psi}_{t}-v \nabla_{L}^{2} \tilde{\psi}=0, \\
\tilde{p} / \rho=\tilde{\varphi}_{t}-g \widetilde{z} .
\end{array}\right\}
$$

Here we can write for the normalized solutions:

$$
\begin{aligned}
\tilde{\varphi}= & \frac{\varepsilon}{2 \cosh k H}[\exp \{\kappa(c+H)\} \\
& \left.+A_{1} \exp \{-\kappa(c+H)\}\right] \exp (i \kappa a+n t), \\
\tilde{\psi}= & \frac{\varepsilon}{\cosh k H}\left[B_{1} \exp m c\right. \\
& \left.+B_{2} \exp \{-m(c+H)\}\right] \exp (i \kappa a+n t) .
\end{aligned}
$$

Here $\varepsilon$ is given by

$\varepsilon=\omega \eta_{0} /(k \tanh k H)$,

where $\eta_{0}$ is the initial wave amplitude. In (19)-(20) we have defined the complex quantities

$$
\left.\begin{array}{l}
\kappa=k+i \alpha \\
n=-\beta-i \omega \\
m^{2}=\kappa^{2}+n / \nu
\end{array}\right\}
$$

where $k, \omega$ are the real and positive wave number and wave frequency, respectively, and $\alpha, \beta$ are real growth/attenuation rates in space and time. Jenkins (1986) was the first to include the combined spatial and temporal modulation of the wave amplitude in Lagrangian wave drift calculations for the deep water case. Assuming that $\left|\kappa^{2}\right| \ll|n / \nu|$, we obtain

$$
m=(1-i) \gamma, \quad \gamma \equiv[\omega /(2 v)]^{1 / 2}
$$

Here $1 / \gamma$ is the thickness of the thin viscous boundary layers (the vorticity layers) at the top and the bottom of our model. We take that $H \gg 1 / \gamma$, which will always be well fulfilled in any practical application. 
From the bottom boundary condition (16) we obtain

$i \kappa\left(1+A_{1}\right)+2 m B_{1} \exp (-m H)-2 m B_{2}=0$,

$\kappa\left(1-A_{1}\right)-2 i \kappa B_{1} \exp (-m H)-2 i \kappa B_{2}=0$.

We assume that the fluctuating tangential wind stress is negligible compared to the fluctuating normal component (Phillips, 1977). Hence, we take $\tilde{\tau}_{1}=0$ in (14). We then find

$i \kappa^{2}\left[\exp (\kappa H)-A_{1} \exp (-\kappa H)\right]+\left(m^{2}-\kappa^{2}\right)\left[B_{1}+B_{2} \exp (-m H)\right]=0$.

In this analysis we assume that the growth/decay rates are small, i.e.

$\left.\begin{array}{l}|\alpha| / k \ll 1,|\beta| / \omega \ll 1 \\ \cosh \alpha H \approx 1, \quad \sinh \alpha H \approx \alpha H\end{array}\right\}$

and that the order of the small quantities are such that $O(|\alpha| / k) \sim O(|\beta| / \omega)$. We then easily determine $A_{1}, B_{1}, B_{2}$ from (24)-(26). The results for the potential and the vorticity-part of the wave field become:

$$
\begin{aligned}
\tilde{\varphi}= & \frac{\varepsilon}{\cosh k H}[\cosh \{\kappa(c+H)\} \\
& \left.+\frac{\kappa}{m} \exp \{-\kappa(c+H)\}\right] \exp (i \kappa a+n t), \\
\tilde{\psi}= & \frac{i \kappa \varepsilon}{m \cosh k H}\left[-\frac{2 \kappa}{m} \sinh \kappa H \exp m c\right. \\
& \left.+\left(1+\frac{\kappa}{m}\right) \exp \{-m(c+H)\}\right] \exp (i \kappa a+n t) .
\end{aligned}
$$

We allow the waves in our problem to be supported by a suitably arranged small normal wind stress $\tilde{\sigma}$ at the surface. Introducing the potential and vorticity parts $\tilde{\varphi}$ and $\tilde{\psi}$, respectively, we find from (14) that

$$
-n \tilde{\sigma} / \rho=n^{2} \tilde{\varphi}+g \tilde{\varphi}_{c}+2 v n \tilde{\varphi}_{c c}-g \tilde{\psi}_{a}-2 v n \tilde{\psi}_{a c}, \quad c=0
$$


This yields the dispersion relation in complex form for our problem. We here define the normal wind stress as:

$\tilde{\sigma} / \rho=-\varepsilon \delta \exp (i \kappa a+n t)$

By inserting from (28), (29) and (31) into (30), applying (26) and (27), we find from the real and imaginary parts of (30) that to lowest order

$\omega=[g k \tanh k H]^{1 / 2}$,

and

$$
\begin{aligned}
\delta= & \frac{\left(\omega^{2}+g k\right) \exp (-k H)}{2 \omega \cosh k H}(k / \gamma)+2 \omega\left(k^{2} / \gamma^{2}\right) \\
& -2 \beta+\left[\left(\omega^{2} H-\mathrm{g}\right) \tanh k H-g k H\right](\alpha / \omega) .
\end{aligned}
$$

By applying (32), we note that (33) can be written as

$$
\delta=\frac{\omega}{\sinh 2 k H}(k / \gamma)+2 \omega\left(k^{2} / \gamma^{2}\right)-2 \beta-2 C_{g} \alpha
$$

Here $C_{g}=d \omega / d k$ is the group velocity. It is related to the phase speed $C=\omega / k$ by the formula (Lamb, 1932)

$C_{g}=\frac{1}{2} C\left(1+\frac{2 k H}{\sinh 2 k H}\right)$

From (34) we note that the normal wind stress along the undulating surface may sustain the wave against dissipation. When $k H \rightarrow \infty$, the first term on the right-hand side of (34) vanishes, and the relation becomes equal to the one obtained by Jenkins (1986) for deep-water waves. Negative values of $\alpha, \beta$ mean wave growth by our definition, and that is possible if the normal wind stress amplitude in phase with the wave slope satisfies

$$
\delta>\frac{\omega}{\sinh 2 k H}(k / \gamma)+2 \omega\left(k^{2} / \gamma^{2}\right)
$$


In shallow water the first term on the right-hand side of (34) dominates. Without any influence from the wind, and purely spatial decay $(\delta=\beta=0)$, we obtain for the spatial damping rate that $\alpha=k /(4 \gamma H)$. For purely temporal decay $(\delta=\alpha=0)$, we find $\beta=$ $\omega /(4 \gamma H)$, or $\beta=C_{g} \alpha$, since here $C_{g}=\omega / k$. As first pointed out by Gaster (1962), this result is generally valid for problems involving weak spatial or temporal amplitude variations.

\section{Conservation of mean wave momentum and mean wave energy}

The relation (34) leads to an interesting nonlinear result for the conservation of wave momentum. We define the form stress $\tau_{D}$ (often referred to as the form drag) at the surface as

$\tau_{D}=-\overline{\tilde{\sigma} \tilde{z}_{a}}, \quad c=0$,

where we insert real parts of the normal stress and the surface slope. The over-bar denotes averaging over one wave cycle. Utilizing (31), we then find

$\tau_{D} / \rho=\frac{\omega \delta}{2 \tanh k H} \eta_{0}^{2} \exp (-2 \alpha a-2 \beta t)$

The Stokes drift $u^{(S)}$ for this problem can be written (Longuet-Higgins, 1953):

$u^{(S)}=\frac{\omega k \cosh 2 k(c+H)}{2 \sinh ^{2} k H} \eta_{0}^{2} \exp (-2 \alpha a-2 \beta t)$.

The total wave momentum $U^{(S)}$ per unit density thus becomes

$U^{(S)}=\int_{-H}^{0} u^{(S)} d c=\frac{\omega}{2 \tanh k H} \eta_{0}^{2} \exp (-2 \alpha a-2 \beta t)=E /(\rho C)$,

where $E$ is the total wave energy (Starr, 1959; Phillips, 1977). From (38) and (40) we find for the form stress that 
$\tau_{D}=\delta \rho U^{(S)}$.

Another fundamental quantity, called the virtual wave stress $\tau_{w}$, appears from the two first terms on the right-hand side of (34). Multiplying by $\rho U^{(S)}$, we can write the virtual wave stress as

$\tau_{w}=\left[\frac{\omega}{\sinh 2 k H}(k / \gamma)+2 \omega\left(k^{2} / \gamma^{2}\right)\right] \rho U^{(S)}$

The role of $\tau_{w}$ is to redistribute mean momentum from waves to Eulerian currents when wave energy is lost in the dissipation process. This can be seen as follows: For temporally damped waves, and no wind, i.e. $\alpha=\delta=0$, we realize from (34) and (42) that $\tau_{w}=2 \beta \rho U^{(S)}$.

Hence:

$\int_{0}^{\infty} \tau_{w} d t=\rho U^{(S)}(t=0)$

The right-hand side is the total initial wave momentum, and accordingly, all the mean wave momentum is transferred to ocean currents by the action of the virtual wave stress. This was first shown by Longuet-Higgins (1969) for deep water waves $(k H \gg 1)$. We have here demonstrated that this also applies to water of finite depth.

Multiplying (34) by $U^{(S)}$ from (40), and utilizing the results (41) and (42), we obtain:

$\partial M / \partial t+\partial\left(C_{g} M\right) / \partial a=\tau_{D}-\tau_{w}$,

where $M=\rho U^{(S)}$ is the total wave momentum. The relation (44) constitutes a conservation equation for the mean wave momentum in a single wave, where $C_{g} M$ is the advection of mean wave momentum by the group velocity, the form stress $\tau_{D}$ is the momentum flux from the atmosphere into the wave field, and the virtual wave stress $\tau_{w}$ is the momentum flux from the waves into Eulerian ocean currents. We note that (44) is a variant of the well-known wave 
action equation. The spatially two-dimensional (Eulerian) form of (44) is routinely solved by numerical wave prediction models; see e.g. Komen et al. (1994). In general, (44) should also contain a non-linear wave-wave interaction term that distributes energy between different wave components. It vanishes here since we only look at one component, represented by the dominant peak of the wave spectrum. However, the wave-wave interaction term is small for a saturated sea state, and vanishes when integrated over all wave components; see e.g. Röhrs et al. (2012).

Utilizing that $E=C M$, we can write (44) as a wave energy balance:

$\partial E / \partial t+\partial\left(C_{g} E\right) / \partial a=C \tau_{D}-C \tau_{w}$

Here $C_{g} E$ is the advection of mean wave energy by the group velocity, $C \tau_{D}$ represents the work by the wind on the waves, and $C \tau_{w}$ is the dissipation due to friction in the fluid.

The equations (44) and (45) for the conservation of mean wave momentum and mean wave energy in a layer of finite depth have been derived by Weber et al. (2008) in the case of linear (Rayleigh) friction. The derivation here in Lagrangian form for a Newtonian viscosity appears to be novel.

\section{The Lagrangian horizontal mean flow and the divergence effect}

The horizontal mean drift velocities are obtained from (11) and (12). To simplify, we introduce the complex horizontal mean velocity as

$W \equiv \bar{x}_{t}+i \bar{y}_{t}$

We then obtain 


$$
\begin{aligned}
W_{t}+i f W-v \nabla_{L}^{2} W= & -(\bar{p} / \rho+g \bar{z})_{a}-\overline{J(\tilde{p} / \rho, \tilde{z})} \\
- & v\left(2 \overline{\tilde{x}_{a} \tilde{x}_{t a a}}+2 \overline{\tilde{z}_{c} \tilde{x}_{t c c}}+2 \overline{\left(\tilde{z}_{a}+\tilde{x}_{c}\right) \tilde{x}_{t a c}}\right. \\
& \left.+\overline{\tilde{x}_{t a} \nabla_{L}^{2} \tilde{x}}+\overline{\tilde{x}_{t c} \nabla_{L}^{2} \tilde{z}}\right)
\end{aligned}
$$

where we insert the real parts of $\tilde{x}, \tilde{z}, \tilde{p}$ for the primary wave motion on the right-hand side of this equation.

By averaging over the wave cycle, we realize that for temporally/spatially modulated waves, (9) can be fulfilled only when both sides are identically zero. Accordingly, for the continuity to second order

$\bar{x}_{a}+\bar{z}_{c}+\overline{J(\tilde{x}, \tilde{z})}=0$.

By integrating in the vertical, utilizing that $\bar{z}(c=-H)=0$, we obtain from (48) that

$$
h^{(L)} \equiv \bar{z}(c=0)=-\int_{-H}^{0} \bar{x}_{a} d c-\int_{-H}^{0} \overline{J(\tilde{x}, \tilde{z})} d c,
$$

where $h^{(L)}$ is the Lagrangian mean displacement of the surface level due to waves.

From an order of magnitude estimate, the acceleration and the viscous force can be neglected in (13) to second order; see also Weber et al. (2008). Hence for the mean vertical balance to $O\left(\varepsilon^{2}\right)$ :

$$
(\bar{p} / \rho+g \bar{z})_{c}=-\overline{J(\tilde{x}, \tilde{p} / \rho)}-g \overline{J(\tilde{x}, \tilde{z})}
$$

Integrating (50) in the vertical, and using that $\bar{p}(c=0)=0$ at the free surface, together with (49), we find that

$$
(\bar{p} / \rho+g \bar{z})_{a}=g h_{a}^{(L)}+\int_{c}^{0}\left\{{\overline{J(\tilde{x}, \tilde{p} / \rho)_{a}}}+g \overline{J(\tilde{x}, \tilde{z})}_{a}\right\} d c^{\prime}
$$

Applying the potential part (28) of the linear solution, we find for the horizontal mean pressure forcing in (47); see also Weber et al. (2008): 


$$
\begin{aligned}
(\bar{p} / \rho+g \bar{z})_{a}+\overline{J(\tilde{p} / \rho, \tilde{z})}= & -g \int_{-H}^{0} \bar{x}_{a a} d c \\
& +\frac{1}{H}\left(C_{g} / C-1 / 2\right) C U_{a}^{(S)}-u_{t}^{(S)}
\end{aligned}
$$

Since the total wave energy $E$ is given by $E=C \rho U^{(S)}$ (Starr, 1959; Phillips, 1977), we realize that the second term on the right-hand side is equal to the radiation stress component $S_{22}$ per unit depth for waves along the 1-axis of Longuet-Higgins and Stewart (1960). We note that the term proportional to $S_{22}$ does not vary in the vertical. Hence a vertically-varying radiation stress does not occur as forcing in a pure Lagrangian formulation of the drift problem. This is contrary to the results of Mellor $(2003,2005)$, who applies a coordinate transformation for the vertical coordinate and finds vertically-varying radiation stress terms in the equations for the mean flow. It should be noted that in deep water, where $C_{g}=C / 2$, the radiation stress term in (52) vanishes identically.

Finally, by inserting for the divergence term in (49), we obtain for the Lagrangian mean surface elevation that

$h^{(L)}=-\int_{-H}^{0} \bar{x}_{a} d c+U^{(S)} / C$

The nonlinear displacement of the Lagrangian mean water level (53) is different from the nonlinear Eulerian mean water level (Longuet-Higgins, 1986, 1988). Denoting the latter by $h^{(E)}$, we obtain by integrating the Eulerian continuity equation $u_{x}^{(E)}+w_{z}^{(E)}=0$ in the vertical from the bottom to the surface $z=\eta$, that

$h_{t}^{(E)}=-\left(\overline{\int_{-H}^{\eta} u^{(E)} d z}\right)_{x} \approx-\int_{-H}^{0} \bar{x}_{t a} d c$

correct to second order. Hence, by integrating in time, and inserting into (53), we find that

$h^{(L)}=h^{(E)}+U^{(S)} / C$. 
We can compare this result with Longuet-Higgins (1988), who basically used the Bernoulli equation to obtain the difference between the Lagrangian and the Eulerian mean surface elevation in irrotational inviscid surface waves in water of finite depth. In our notation (Longuet-Higgins' eqn. (3.10)), the difference can be expressed as

$h^{(L)}-h^{(E)}=\frac{1}{2 g}\left[C u^{(S)}(c=0)+\overline{\tilde{u}}^{2}(c=-H)\right]$.

The Stokes drift is given generally by (39). Accordingly, in (56) we insert for non-damped waves at the surface

$u^{(S)}(c=0)=\frac{\omega k \cosh 2 k H}{2 \sinh ^{2} k H} \eta_{0}^{2}$.

Furthermore, from elementary wave theory (LeBlond and Mysak, 1978):

$\overline{\tilde{u}^{2}}(c=-H)=\frac{\omega^{2}}{2 \sinh ^{2} k H} \eta_{0}^{2}$,

where $\omega$ is given by (32). By inserting from (57) and (58) into (56), we find that the righthand side becomes $U^{(S)} / C$, and thus we recover our result (55) for finite depth obtained from a Lagrangian analysis. The fact that the Lagrangian mean surface level is higher than the Eulerian mean level is often referred to as the divergence effect after McIntyre (1988), who related this effect in deep water waves to the fact that the continuity equation for the mean variables in Lagrangian form is divergent; see (48). For finite depth, the difference between the Lagrangian and the Eulerian mean level has been calculated by $\mathrm{Ng}$ (2004b) for partially standing waves. For progressive waves in an inviscid fluid the Lagrangian mean surface elevation has been calculated to third order by Clamond (2007) and Chen et al. (2010).

In all our problems with a weakly-damped mean flow, we can safely assume that $\left|W_{c c}\right| \gg\left|W_{a a}\right|$. Utilizing (52) and (53), (47) can then be written as

$$
\begin{aligned}
v W_{c c}-W_{t}-i f W= & g h_{a}^{(L)}-\frac{1}{H}\left[C_{0}^{2} / C+C / 2-C_{g}\right] U_{a}^{(S)} \\
& -u_{t}^{(S)}+v\left(F_{p}+F_{b l}\right) .
\end{aligned}
$$


Here $F_{p}$ denotes the nonlinear wave interaction terms from the potential part of the wave solution, and $F_{b l}$ the viscous boundary-layer terms. These terms will be specified in the various examples that follow. Furthermore, $C_{0}=(g H)^{1 / 2}$ is the shallow-water wave speed in a non-rotating ocean.

\section{Surface waves in deep water}

When $k H \gg 1$, we have surface waves in deep water, and the drift problem is considerably simplified. In this case the Stokes drift (39) becomes

$u^{(S)}=u_{0} \exp (2 k c-2 \alpha a-2 \beta t)$

where

$u_{0}=\eta_{0}^{2} \omega k$

\subsection{Permanent waves}

We here consider waves that propagate with constant amplitude, i.e. $\alpha=\beta=0$ in (34), and hence $\delta=2 \omega k^{2} / \gamma^{2}$. In this case we assume that the waves are so long that we safely can neglect the effect of surface films. Taking that $\eta=\eta_{0} \sin (k a-\omega t)$ for the permanent wave, we obtain from (31) for the fluctuating normal stress (Weber, 1983b):

$\tilde{\sigma}=-4 \rho v \eta_{0} \omega k \cos (k a-\omega t), \quad c=0$,

i.e. maximum negative wind stress where the wave slope has its largest positive value (at the rear of the crest). Here the vertical wave velocity is negative, so the external force and the displacement is in the same direction, which yields a positive energy input to the waves (it is 
the same in the front, where both contributions are positive). The form stress (38) in this case is positive, and given by $\tau_{D}=2 \rho v \eta_{0}^{2} \omega k^{2}$.

Equation (59) for the steady mean flow now reduces to (Weber, 1983b):

$v W_{c c}-i f W=v k^{2} u_{0}\left[4 \exp (2 k c)-4 \frac{\gamma}{k} \exp (\gamma c)(\cos \gamma c-\sin \gamma c)\right]$.

Here $\gamma$ is the large parameter defined by (23). We take that the wind is directed along the waves $\left(\tilde{\tau}_{2}=\bar{\tau}_{2}=0\right)$. By assuming a constant mean wind stress $\bar{\tau}_{1}=\tau$ in the $X$-direction, the boundary conditions become from (15):

$W_{c}=-2 k u_{0}+T, c=0$,

where $T=\tau /(\rho v)$. In the deep ocean we require

$W \rightarrow 0, \quad c \rightarrow-\infty$.

If we neglect the effect of the earth's rotation, we note that the first term on the right-hand side of (63) yields the classic Stokes drift (60) for deep water. We also recall that in the absence of wind-forcing, we must have $W_{c}=0$ at the free surface (Longuet-Higgins, 1953). The first term on the right-hand side of (64) is due to the presence of the undulating normal stress (62) at the moving surface (Weber, 1983b). We note that the second term in (64), together with the homogeneous part of (63), yields the classic Ekman solution $W^{(T)}$ for an infinitely deep ocean, where the surface current is directed 45 degrees to the right of the wind in the northern hemisphere. We can write the solution of (63):

$W=W^{(T)}+W^{(w)}$,

where the wave-induced part of the solution is denoted by $W^{(w)}$. Introducing the Ekman depth $D=(2 v / f)^{1 / 2}$, the Stokes depth $L=1 /(2 k)$, and the friction velocity in the water $u_{*}=(\tau / \rho)^{1 / 2}$, the well-known Ekman solution becomes $W^{(T)}=\frac{2}{(1+i) f D} u_{*}^{2} \exp ((1+i) c / D)$

while the wave-induced part of the drift solution can be written 


$$
\begin{aligned}
W^{(w)}= & u_{0}\left[q \exp (c / L)+\frac{D}{(1+i) L}(1-q) \exp ((1+i) c / D)\right. \\
& \left.-2 k \gamma^{-1} \exp (\gamma c)(\cos \gamma c+\sin \gamma c)\right] .
\end{aligned}
$$

Here $q=1 /\left(1-2 i L^{2} / D^{2}\right)$. Taking $v=5 \times 10^{-2} \mathrm{~m}^{2} \mathrm{~s}^{-1}$, which is a reasonable bulk value for the turbulent surface layer, and $f=1.2 \times 10^{-4} \mathrm{~s}^{-1}$, we have in Fig. 1 plotted the dimensionless wave drift current $W^{(w)} / u_{0}$ from (68) as function of dimensionless depth $c / L$, when $\lambda=2 \pi / k=100 \mathrm{~m}$. In this problem $L / D=0.28$.

\section{(Figure 1)}

We note from the figure that the effect of the Coriolis force reduces the wave drift at the surface, and causes a veering to the right. The deflection angle to the right of the wave propagation direction in this example is about 13 degrees, and the magnitude of surface current is $0.72 u_{0}$. The non-dimensional vertical gradient $\left(L / u_{0}\right) \partial u^{(w)} / \partial c$ at the surface is given by -1 from (64), but this is not well resolved in the plot.

Relating the wind speed $U_{10}$ at $10 \mathrm{~m}$ height to the wave field, using that the phase speed $C$ of the dominant component in the surface wave spectrum approximately satisfies $C \sim U_{10}$ (Phillips, 1977), and applying a realistic model for the bulk eddy viscosity in the surface layer caused by wind, it is found that the magnitudes of surface Ekman current (67) and the waveinduced drift current (68) are of the same order of magnitude (Weber, 1983b).

\subsection{Temporally attenuated waves}

As mentioned in Section 1, we can write the Lagrangian mean drift $u=\bar{x}_{t}$ in periodic wave motion with weak viscosity as the sum of the Stokes drift $u^{(S)}$, and an Eulerian mean current caused by the effect of friction (Longuet-Higgins, 1953). It is convenient to separate the Eulerian mean current into two parts; one part $u^{(v)}$ which is confined to a very thin 
vorticity boundary layer near the surface, and one quasi-Eulerian part $u^{(E)}$ which diffuses downward from the surface and modifies the drift current in the interior (Jenkins, 1986). For the complex drift velocity in a rotating ocean we can write:

$W=W^{(S)}+W^{(E)}+W^{(v)}$.

For temporally damped gravity waves and constant eddy viscosity, (59) reduces to (Weber, 1983a):

$$
\begin{aligned}
v W_{c c}-W_{t}-i f W & =v u_{0} k^{2}[8 \exp (2 k c)) \\
& \left.-4 \gamma k^{-1} \exp (\gamma c)(\cos \gamma c-\sin \gamma c)\right] \exp (-2 \beta t) .
\end{aligned}
$$

Here, from (34) with $\delta=\alpha=0$, we have $\beta=2 v k^{2}=\omega k^{2} / \gamma^{2}$ (Lamb,1932). The boundary conditions are:

$\left.\begin{array}{l}W_{c}=0, c=0 \\ W=0, c \rightarrow-\infty\end{array}\right\}$

The initial condition of the mean drift problem is not so obvious, since the motion is not started from rest. Stokes' classic solution for the wave drift is derived from the irrotational wave field. When the wave field is established, a Stokes drift instantaneously results. The effects on the mean mass transport induced by viscosity and the earth's rotation manifest themselves on a much longer time scale. Therefore, we take that the mass transport velocity initially coincides with the Stokes drift, i.e.

$W(t=0)=u_{0} \exp (2 k c)$

By definition we take that in (69)

$W^{(S)}=u_{0} \exp (2 k c-2 \beta t)$.

Then from (70):

$W^{(v)}=-u_{0}\left[2 k \gamma^{-1} \exp (\gamma c)(\cos \gamma c+\sin \gamma c)\right] \exp (-2 \beta t)$.

Finally, $W^{(E)}$ is the solution of 
$v W_{c c}^{(E)}-W_{t}^{(E)}-i f W^{(E)}=i f W^{(S)}=i f u_{0} \exp (2 k c-2 \beta t)$.

From (71) the surface boundary condition for $W^{(E)}$ becomes

$W_{c}^{(E)}=2 k u_{0} \exp (-2 \beta t), c=0$.

As explained earlier, Longuet-Higgins (1969) introduced the concept of virtual wave stress in a non-rotating infinitely deep fluid in order to explain the transfer of mean momentum from decaying waves to Eulerian drift currents. In a rotating deep ocean we can write for the virtual wave stress (Weber and Førland, 1990):

$\tau_{w}=\rho v W_{c}^{(E)}(c=0)$.

From (77) we find that $\tau_{w}=2 \rho v k u_{0} \exp (-2 \beta t)$, which conforms to the result (42) in the short wave limit $k H \gg 1$. We note from (77) that $\tau_{w}$ acts as a boundary forcing for the quasiEulerian flow, quite like a time-decaying wind stress in the Ekman case, but now related to the wave field. The virtual wave stress in this case gives rise to a forward drift accompanied by damped inertial oscillation in the fluid (Weber, 1983a), as will be demonstrated below.

Neglecting the small vorticity-layer solution in the initial condition (72), we obtain for the quasi-Eulerian flow:

$W^{(E)}(t=0)=0$

From (75), (76) and (78), utilizing that $W^{(E)}$ must vanish for $c \rightarrow-\infty$, we obtain by Laplace transforms that

$$
\begin{aligned}
W^{(E)} / u_{0}= & -(1-\hat{q}) \exp (2 k c-2 \beta t) \\
& +2 k v^{1 / 2}(2-\hat{q}) \exp (-2 \beta t) \int_{0}^{t} \frac{\exp \left\{(2 \beta-i f) \xi-c^{2} /(4 v \xi)\right\}}{(\pi \xi)^{1 / 2}} d \xi \\
& +2 k v^{1 / 2}(1-\hat{q}) \exp (-i f t) \int_{0}^{\infty} \frac{\exp (-\xi t) \cos \left(c \xi^{1 / 2} / v^{1 / 2}\right)}{\pi \xi^{1 / 2}\left(\xi+4 k^{2} v\right)} d \xi
\end{aligned}
$$


Here $\hat{q}=1 /\left(1-i L^{2} / D^{2}\right)$ (Weber, 1998). In Fig. 2 we have displayed the surface hodograph of the non-dimensional Lagrangian mean drift current $W=W^{(S)}+W^{(E)}$, scaled by $u_{0}$. The vorticity-layer part (74) is here negligibly small. The waves propagate along the $x$-axis with $\lambda=100 \mathrm{~m}$, and we have taken that $f=10^{-4} \mathrm{~s}^{-1}$.

\section{(Figure 2)}

We infer from Fig. 2 that we have a net wave-induced displacement of particles to the right of the of the wave propagation direction for a viscous fluid, accompanied by damped inertial oscillations; see also Weber (1983a). The inertial period in this example is $T_{i}=2 \pi / f=$ 17.5 $\mathrm{h}$. We note from the figure that after about 5 inertial periods, only inertial oscillations prevail. At that time a single particle has travelled about 4.2 inertial radii $\left(=u_{0} / f\right)$ from the origin, and the smoothed trajectory is deflected a little less than 40 degrees to the right of the wave propagation direction. It is worth remarking the fundamental difference between solutions obtained with $v=0$ (blue circle) and with $v \neq 0$ (red curve).

\subsection{Effect of a vertically-varying eddy viscosity}

In the oceanic upper layer the turbulent mixing varies in the vertical direction; basically increasing downwards from the surface. In Weber and Melsom (1993) the linear wave field was subject to a small constant eddy viscosity $v_{0}$, while the eddy viscosity that redistributes the mean momentum was, by analogy with the classic result for turbulent flow over a flat plate, taken to vary linearly with depth (Madsen, 1977). The eddy diffusivity for the mean flow can then be written

$v=v_{0}-\kappa_{K} u_{*} c$, 
where $\kappa_{K}$ is Von Kármán's constant $(\approx 0.4)$ and $u_{*}=(\tau / \rho)^{1 / 2}$ is the friction velocity in the water. This is a reasonable assumption when the Stokes depth $L=1 /(2 k)$ is smaller than the mixed-layer thickness at the surface, which is usually the case for wind-generated surface waves. The equation for the quasi-Eulerian part of the solution then becomes (Weber and Melsom, 1993):

$$
\begin{aligned}
v W_{c c}^{(E)}-\kappa_{K} u_{*} W_{c}^{(E)}-W_{t}^{(E)}-i f W^{(E)}= & u_{0}\left[2 k \kappa_{K} u_{*}(2 k c+1)\right. \\
& +i f] \exp (2 k c-2 \beta t)
\end{aligned}
$$

The surface boundary condition becomes

$W_{c}^{(E)}=2 k u_{0} \exp (-2 \beta t), \quad c=0$,

while

$W^{(E)} \rightarrow 0, c \rightarrow-\infty$

The importance of turbulent wind stress on wave growth is pointed out among others by Chalikov and Makin (1990). For turbulent air flow above the waves, the temporal wave growth rate can be obtained from the asymptotic analyses by Knight (1977) and Jacobs (1987). In our notation Knight's result can be written:

$\beta / \omega=\kappa_{K} u_{*} s^{1 / 2}\left(C-U_{\text {wind }}\right) / C^{2}+k^{2} / \gamma^{2}$,

where $s=\rho_{\text {air }} / \rho$ is the density of air $\rho_{\text {air }}$ relative to that of water, and $U_{\text {wind }}$ is the wind speed at height $1 / k$ above the water. We note that the last term on the right-hand side of (84) is the previous result for deep water waves (swell) propagating in calm conditions. We notice that for adverse winds $\left(U_{\text {wind }}<0\right)$, the damping rate can increase considerably.

As an example we take that the waves are traveling against the wind. For a wavelength 
$\lambda=100 \mathrm{~m}$, and a friction velocity $u_{*}=0.016 \mathrm{~m} \mathrm{~s}^{-1}$ (adverse wind speed $U_{10}$ of about

$12 \mathrm{~m} \mathrm{~s}^{-1}$ ), we find $\beta=3 \times 10^{-5} \mathrm{~s}^{-1}$ (Weber and Melsom, 1993). From Bye (1988) the small "background" eddy viscosity is estimated as $v_{0}=1.4 \kappa_{K} u_{*}^{3} /(s g)$. Taking the initial wave amplitude $\eta_{0}=1 \mathrm{~m},(81)$ was solved numerically, subject to (82), (83), and the initial condition (78). In Fig. 3 we have displayed the wave-induced current $W^{(w)}=W^{(S)}+W^{(E)}$, where $W^{(S)}$ is given by (73). The result is non-dimensionalized by the friction velocity.

\section{(Figure 3)}

We note from the figure that a depth-dependent eddy viscosity promotes a more rapid decrease of the surface current with time (solid line), as well as leading to a stronger suppression of the inertial part of the mean motion. Also, we find that that the surface current is more aligned along the wave propagation direction (upward in the plot) than for constant eddy viscosity. In the same way, an eddy viscosity that increases with depth causes the winddriven Ekman surface current to be aligned closer to the wind direction (Madsen, 1977; Jenkins, 1987; Weber and Melsom, 1993).

\subsection{Spatially attenuated waves}

We note from (77) that the virtual wave stress is decaying in time for temporally damped waves. Hence the quasi-Eulerian current will finally vanish, as seen in Figs. 2 and 3. This alters when we have spatially damped waves. Such waves may for example occur when we operate a wave maker at the end of a laboratory tank, generating frequency-forced waves. Now, at every position down the tank, the virtual wave stress is independent of time, promoting a drift current that increases in time. Using the sub-division (69) of the Lagrangian mean velocity, and neglecting the Coriolis force as well as the effect of air above the water, 
the virtual wave stress relevant for wave tank experiments can be written (Weber, 2001):

$\tau_{w}=\rho v u_{c}^{(E)}(c=0)=2 \rho v k u_{0} \exp (-2 \alpha a)$

where $\alpha=4 k^{3} v / \omega$ (Jenkins, 1986). In this case the Lagrangian mean drift solution at the surface becomes

$u(c=0)=u_{0}\left[1+4\left(v k^{2} t / \pi\right)^{1 / 2}\right] \exp (-2 \alpha a)$

We thus notice that for $t>0$, the Lagrangian surface drift always will be larger than the Stokes drift at the surface. With $\tau_{D}=\beta=0$, we find from (42) for spatially-decaying short gravity waves that

$\tau_{w}=-\left(C_{g} M\right)_{a}$,

where $C_{g}$ is the group velocity, and $M=\frac{1}{2} \rho \omega \eta_{0}^{2} \exp (-2 \alpha a)$ is the mean wave momentum for this problem. Here the term $\left(C_{g} M\right)_{a}$ is recognized as the nonzero component of the divergence of the radiation stress tensor of Longuet-Higgins and Stewart (1960) in deep water, now in Lagrangian coordinates.

\section{Effect of surface films on drift in water waves}

It is well known that surface films of biogenic origin may cover large areas of the ocean surface (Gade et al., 2006). In addition to these natural films, we find pollutant organic slicks from petroleum spills or municipal effluents. Such films resist the formation of windgenerated capillary waves, and also strongly enhance the attenuation of short gravity waves (Dorrestein, 1951). In the presence of surface films, the virtual wave stress is greatly enhanced, leading to Eulerian mean currents that become stronger than the Stokes drift in a relatively short time interval; see e.g. Weber and Førland (1989), Weber and Saetra (1995), Christensen and Weber (2005a). The large increase in surface drift in the presence of an 
inextensible film was originally demonstrated by Craik (1982) from vorticity considerations.

Floating layers of finite thickness may also appear at the ocean surface. The wave damping in such cases has been studied among others by Jenkins and Jacobs (1997), Jenkins and Dysthe (1997) and De Carolis et al. (2005).

We here consider the effect of a thin (monomolecular) elastic film. The surface tension $S$ depends on the concentration of surfactant $\Gamma$ in the film, which is assumed to vary slightly around an equilibrium value $\Gamma_{0}$. For the linear motion we may write $S=S_{0}+\tilde{S}$, where $S_{0}=S\left(\Gamma_{0}\right)$. The boundary condition for the linear problem, including the effect of surface tension, can be written (Weber and Saetra, 1995):

$\left.\begin{array}{l}\tilde{\tau}_{1}=\rho v\left(\tilde{x}_{t c}+\tilde{z}_{t a}\right)-\tilde{S}_{a} \\ \tilde{\sigma}=-\tilde{p}+2 \rho v \tilde{z}_{t c}-S_{0} \tilde{z}_{a a} \\ \tilde{S}=\rho E_{*} \tilde{x}_{a}\end{array}\right\}, c=0$.

Here $E_{*}$ is the surface dilational modulus per unit density; see Lucassen (1968).

For waves in the absence of wind $\left(\tilde{\tau}_{1}=\tilde{\sigma}=0\right)$ in (88), the extreme case $E_{*} \rightarrow \infty$ corresponds to the inextensible film limit (Lamb, 1932). In that case the dynamic boundary condition in the horizontal direction reduces to $\tilde{x}=0$ at the film-covered surface.

Accordingly, to first order, the film only moves vertically in the inextensible limit. However, for a freely floating film, the wave-induced horizontal second-order Lagrangian velocity at the surface (and the horizontal motion of the film) will be different from zero. Again, writing the Lagrangian mean flow as $u=\bar{x}_{t}$, the governing equation in the inextensible limit becomes (Christensen and Weber, 2005a):

$$
\begin{aligned}
v u_{c c}-u_{t}= & v k^{2} u_{0}\left[4 \exp (2 k c)+\gamma^{2} k^{-2}\{3 \exp (2 \gamma c)\right. \\
& +4 \exp (\gamma c) \sin \gamma c\}] \exp (-2 \alpha a),
\end{aligned}
$$

where now $\alpha=k^{2} /(2 \gamma)$. This equation (including the effect of the earth's rotation) was actually first derived by Weber (1987) for wave-induced flow under grease ice in the marginal 
ice zone, but it also applies to cases where the surface is covered by a thin inextensible film.

If the film (with negligible mass) can move freely along the surface, it can be shown that (Weber and Førland, 1989):

$u_{c}=0, c=0$.

Again, we subdivide the solution as in (69), where the Stokes drift is given by (60) with $\beta=0$. The vorticity-layer solution becomes

$u^{(v)}=u_{0}\left[\frac{3}{4} \exp (2 \gamma c)-2 \exp (\gamma c) \cos \gamma c\right] \exp (-2 \alpha a)$

Hence we find for the virtual wave stress in this case

$\tau_{w}=\rho v u_{c}^{(E)}(c=0)=\frac{1}{2} \rho v \gamma u_{0} \exp (-2 \alpha a)$.

By comparing with the free surface case, e.g. (85), we note that $\tau_{w}$ in (92) is $\gamma / k$ times larger than for a free surface. Since $\gamma / k \gg 1$, and $\tau_{w}$ is independent of time, the increase of the quasi-Eulerian drift current $u^{(E)}$ with time will be quite rapid. In consequence, the Lagrangian drift velocity very soon becomes (much) larger than the Stokes drift. This theory has been applied to calculate the transient wave-induced drift of floating plastic sheets (Christensen and Weber, 2005a), and the fit with laboratory wave tank measurements (Law, 1999) is very good. The same theory can be used for determining the drift of large, flexible floating objects (Christensen and Weber, 2005b). In this paper results are obtained for constant as well as vertically-varying eddy viscosity.

One would intuitively think that the presence of an inextensible surface film should maximize the damping rate of capillary-gravity waves. However, this is not the case. When we have a film with a finite value of $E_{*}$, we can have nearly-critically damped longitudinal dilational waves in the film. They will act as a sink of energy, enhancing the damping of the 
capillary-gravity wave (Dorrestein, 1951).

We consider the effect of elastic films for temporally damped waves. With $\tilde{\tau}_{1}=\tilde{\sigma}=0$, i.e. disregarding the viscous effect of air, the frequency in this problem is

$\omega^{2}=g k+S_{*} k^{3}$

where $S_{*}=S\left(\Gamma_{0}\right) / \rho$. For an inextensible film we find for the temporal damping rate $\beta_{i}=$ $\omega k /(4 \gamma)($ Lamb, 1932). Defining the non-dimensional parameter

$A_{*}=\gamma k^{2} E_{*} / \omega^{2}$,

we can write for the damping rate for an elastic film (Dorrestein, 1951):

$\beta / \beta_{i}=2 A_{*}^{2} /\left(1-2 A_{*}+2 A_{*}^{2}\right)$

We note that $\left(\beta / \beta_{i}\right)_{\max }=2$ for $A_{*}=1$, i.e. the damping is twice that of an inextensible film. This often referred to as the Marangoni effect, because the physics behind it is related to the existence of longitudinal elastic waves in the film (dilational waves), often referred to as Marangoni waves (Lucassen, 1968). When $A_{*}=1$, the frequency of temporally damped Marangoni waves $\omega_{M}$ is $\omega_{M}=1.09 \omega$, so maximum damping occurs when the frequency of free Marangoni waves nearly coincide with the frequency of transverse capillary-gravity waves (Dysthe and Rabin, 1986). This is often referred to as "negative resonance" as the nearly critically-damped Marangoni waves here act as a sink of energy for the capillarygravity wave. Christensen (2005) demonstrates that in this case $\left(A_{*}=1\right)$, the film and the viscous boundary layer oscillates as an elastic membrane with the frequency $\Omega=$ $\left(E_{*}^{2} k^{4} /(2 v)\right)^{1 / 3}$, which is the frequency of forced non-damped dilational waves (Weber and Christensen, 2003).

The nonlinear wave drift in the elastic case is governed by (Weber and Saetra, 1995): 


$$
\begin{aligned}
v u_{c c}-u_{t}= & v k^{2} u_{0}\left[4\left(1+\beta \gamma^{2} /\left(\omega k^{2}\right)\right) \exp (2 k c)\right) \\
& \left.-4 \gamma^{3} k^{-3}\left\{\left(B_{r}+B_{i}\right) \cos \gamma c-\left(B_{r}-B_{i}\right) \sin \gamma c\right\} \exp (\gamma c)\right) \\
& \left.+6 \gamma^{4} k^{-4}\left(B_{r}^{2}+B_{i}^{2}\right) \exp (2 \gamma c)\right] \exp (-2 \beta t) .
\end{aligned}
$$

Here $B_{r}$ and $B_{i}$ are the real and imaginary parts of the complex coefficient

$B_{*}=\frac{k}{\gamma}\left(\frac{k / \gamma+i A_{*}}{1-(1-i) A_{*}}\right)$

The boundary conditions become

$$
\begin{aligned}
& u_{c}= 3 k u_{0}\left[1-\gamma k^{-1}\left(B_{r}-B_{i}\right)-\gamma^{2} k^{-2} B_{r}\right. \\
&\left.+\gamma^{3} k^{-3}\left(B_{r}^{2}+B_{i}^{2}\right)\right] \exp (-2 \beta t), \quad c=0, \\
& u \rightarrow 0, c \rightarrow-\infty .
\end{aligned}
$$

Again, we subdivide the solution $u=u^{(S)}+u^{(v)}+u^{(E)}$, where, as before, $u^{(S)}=$ $u_{0} \exp (2 k c-2 \beta t)$. The vorticity-layer solution $u^{(v)}$ is easily obtained from (96), leading to a diffusion problem for the quasi-Eulerian current:

$v u_{c c}^{(E)}-u_{t}^{(E)}=0$

subject to

$u_{c}^{(E)}=k u_{0}\left(1-3 \gamma k^{-1}\left(B_{r}-B_{i}\right)+\gamma^{2} k^{-2} B_{r}\right) \exp (-2 \beta t), c=0$,

and

$$
u^{(E)} \rightarrow 0, c \rightarrow-\infty
$$


In essence, the quasi-Eulerian momentum is induced to compensate for the loss of momentum in the decaying Stokes drift. Again, this is achieved by the action of the virtual wave stress, defined by (77). In this case we find from (101):

$\tau_{w} / \rho=v u_{c}^{(E)}=\frac{\omega}{2 \gamma} u_{0}\left(\frac{A_{*}^{2}}{1-2 A_{*}+2 A_{*}^{2}}\right) \exp (-2 \beta t), c=0$

We note that $\tau_{w}$ has a maximum for $A_{*}=1$, i.e. when negative resonance occurs. This means that for small times the growth of $u^{(E)}$ is quite rapid, while for larger times the effect of maximum damping for this value of $A_{*}$ becomes increasingly important. Accordingly, for the time dependence, there will be a tendency toward a more pronounced peak for $u^{(E)}$ when $A_{*}$ is near 1 than for values of $A_{*}$ that are different from this resonance value. This is obvious from Fig. 4, where we have plotted the surface value of $U=u^{(E)} / u_{0}$ for various values of $A_{*}$. The solution is obtained from Laplace transforms of (100)-(102), using the approximate initial condition $u^{(E)}(t=0)=0$.

\section{(Figure 4)}

Another interesting effect of the elasticity is related to the variation of the mean drift current with depth. By inspecting the right-hand side of (96), we can show, without solving the equation, that the drift velocity has a maximum below the surface when $A_{*}>2 / 3$ (Weber and Saetra, 1995). From the definition (94) this happens when

$E_{*}>2 C^{2} /(3 \gamma)$

In the problem discussed here, longitudinal dilational waves extracts energy from short transverse capillary-gravity waves. For a purely horizontal elastic film, only dilational waves prevail. By using a Lagrangian formalism, the nonlinear drift in such waves has been investigated by Weber and Christensen (2003) when the film is situated at the interface between two viscous fluids. The linear problem yields a new dispersion relation for dilational 
(Marangoni) waves. When an oscillating stress in the upper fluids acts to prevent wave damping in a fixed (non-moving) film, the mean drift in the lower fluid caused by nondamped dilational waves is purely negative, i.e. in the opposite direction of the wave propagation direction.

\section{Roll motion in short-crested surface waves}

Craik and Leibovich (1976) developed a theory to explain the existence of Langmuir circulations (horizontal rolls in the ocean surface layer; Langmuir, 1938) based on the nonlinear interaction between a horizontally undulating Stokes drift and a unidirectional shear current (the CL1 mechanism; Faller and Caponi, 1978). Since the Stokes drift (second order in wave steepness) and the shear current are assumed to be of the same order of magnitude, this is actually a fourth order theory. It is not the intention here to discuss the various theories of Langmuir circulations. We only point out that by taking the effect of viscosity into account, the bimodal wave spectrum needed to sustain the CL1 mechanism does by itself produce second order horizontal mean vorticity through wave-wave interactions. This vorticity induces roll motion in the wave propagation direction; see e.g. Weber (1985).

A short outline of this problem is as follows: The $X$-axis is still the main propagation direction, but now we have two monochromatic waves propagating at oblique angles to each other. The wave number is $k$ in the $X$-direction and $l,-l$, respectively, in the $Y$-direction. The frequency for gravity waves in deep water now becomes

$\omega=(g K)^{1 / 2}$

where $K=\left(k^{2}+l^{2}\right)^{1 / 2}$ is the overall wave number. The wave trains here propagate with constant (and equal) amplitude $\eta_{0}$, and the expansion parameter in this case is $\varepsilon=\omega \eta_{0} / K$ 
The results for permanent waves in sub-section 6.1, with an external oscillating vertical stress at the surface, are readily generalized (with a little more algebra) to the horizontally twodimensional case. The sea surface now exhibits a three-dimensional pattern of short-crested waves. Averaging in the $X$-direction, and defining the Lagrangian mean drift velocity components $u=\bar{x}_{t}, v=\bar{y}_{t}, w=\bar{z}_{t}$, we obtain for the mean drift problem (Weber, 1985):

$$
\begin{aligned}
& v \nabla_{L}^{2} u+f v-u_{t}=8 v \eta_{0}^{2} \omega k K^{-2}\left[\left(K^{4}+k^{4} \cos 2 l b\right) \exp (2 K c)\right) \\
& \left.-K \gamma\left(K^{2}+k^{2} \cos 2 l b\right) \exp (\gamma c)(\cos \gamma c-\sin \gamma c)\right] \\
& v \nabla_{L}^{2} v-f u-v_{t}-\Pi_{b}=2 \eta_{0}^{2} \omega^{2} l K^{-2}\left[k^{2} \exp (2 K c)\right) \\
& \left.-K^{3} \gamma^{-1} \exp (\gamma c)(\sin \gamma c+\cos \gamma c)\right] \sin 2 l b, \\
& v \nabla_{L}^{2} w-w_{t}-\Pi_{c}=-2 \eta_{0}^{2} \omega^{2} K^{-1}\left[\left(K^{2}+k^{2} \cos 2 l b\right) \exp (2 K c)\right) \\
& \left.-\left(K^{2}+\left(k^{2}-l^{2}\right) \cos 2 l b\right) \exp (\gamma c) \cos \gamma c\right], \\
& v_{b}+w_{c}=0 .
\end{aligned}
$$

Here $\Pi=\bar{p} / \rho+g \bar{z}$, and now $\nabla_{L}^{2}=\partial^{2} / \partial b^{2}+\partial^{2} / \partial c^{2}$. We have here disregarded any mean pressure gradients in the $X$-direction. By utilizing (110), we can introduce a stream function $\psi$ such that $v=-\psi_{c}, w=\psi_{b}$. Neglecting the effect of the Coriolis force, the steady solution which describes roll motion in a plane perpendicular to the main wave propagation, can be written

$$
\begin{aligned}
\psi= & \eta_{0}^{2} \omega K \gamma^{-1}\left[4 l^{3} K^{-2} \gamma^{-1}\{(1-l c) \exp (2 l c)\right. \\
& -\exp (\gamma c) \cos \gamma c\}-K c \exp (2 l c)] \sin 2 l b
\end{aligned}
$$

A conceptual sketch of the primary wave field and the mean circulation in the cross-wave plane is depicted in Fig. 5. 


\section{(Figure 5)}

In Fig. 6 we have plotted the streamlines from the dimensionless stream function $\psi_{*}=\psi \gamma /\left(\eta_{0}^{2} \omega K\right)$ as function of the dimensionless coordinates $y_{*}=2 l b$ and $z_{*}=2 l c$. For the physical parameters we have taken $\eta_{0}=2 \mathrm{~m}, v=10^{-2} \mathrm{~m}^{2} \mathrm{~s}^{-1}, \lambda=2 \pi / K=30 \mathrm{~m}$, and $k / l=3^{1 / 2}$

\section{(Figure 6)}

The maximum upwelling/downwelling velocities occur at the boundaries between the rolls in this problem. For the present choice of parameters we have maximum velocities $|w| \sim 1 \mathrm{~cm} \mathrm{~s}^{-1}$ at a depth of about $1 / 3$ of the cell width.

The horizontal mean drift velocity can be written as

$$
\begin{aligned}
u= & 2 \eta_{0}^{2} \omega k\left[\exp (2 K c)+\left\{k^{2} K^{-2} \exp (2 K c)\right.\right. \\
& \left.-l K^{-1} \exp (2 l c)\right\} \cos 2 l b \\
& \left.-2 K \gamma^{-1} \exp (\gamma c)(\cos \gamma c+\sin \gamma c)\left(1+k^{2} K^{-2} \cos 2 l b\right)\right] \\
& +O\left(K^{2} \gamma^{-2}\right) .
\end{aligned}
$$

At the surface, neglecting small terms, we find from (112) that

$$
u(c=0)=2 \eta_{0}^{2} \omega k\left[1+\left(\cos ^{2} \theta-\sin \theta\right) \cos y_{*}\right]
$$

where $\cos \theta=k / K, \sin \theta=l / K$ and $y_{*}=2 l b$. We note that at small $\theta$ we have a minimum horizontal drift at the nodes of the primary wave system (e.g. $y_{*}=\pi$ in Fig. 6), while for $\sin \theta>\cos ^{2} \theta \quad\left(\theta>38.2^{\circ}\right)$ we have maximum horizontal surface velocity at the nodes. An extension of this problem was made by Førland (1989), who introduced a verticallyvarying eddy viscosity, and also an alteration of the oscillatory surface wind stress which strengthened the circulation. 
Obviously, the surface boundary conditions have a strong impact on the strength of the roll motion in short-crested waves. This was demonstrated by Melsom (1992) who considered obliquely propagating waves under an ice cover, modelling the ice as a thin elastic plate. In this case there are no-slip conditions for the horizontal velocity components at the ice-water interface. This results in a much stronger horizontal vorticity production than for a free surface; see e.g. Weber (1987) for the marginal ice zone, or Weber and Førland (1989) for an inextensible film. In fact, the velocity in the rolls increases by a factor $\gamma / K$ when compared to the free surface case. But now the waves are sheltered from wind influence, and they attenuate in space from the ice edge and inwards. The attenuation rate is one order of magnitude larger than for a free surface without wind, so the wave energy under ice dissipates much faster than in the open ocean.

\section{Lagrangian mean drift in high-frequency shallow water waves}

We now return to consider monochromatic waves along the $X$-axis. In this section we deal with surface waves in shallow ocean, and we neglect viscous effects at the free surface on the wave field (the no-slip bottom dominates). Assuming $k H \ll 1$, we have that $C=C_{g}=$ $C_{0}=(g H)^{1 / 2}$. Then the mean drift equation (59) becomes:

$$
\begin{aligned}
v W_{c c}-W_{t}-i f W & =g h_{a}^{(L)}-\frac{1}{2 H} C_{0} U_{a}^{(S)}-u_{t}^{(S)}+v u_{c c}^{(S)} \\
& +\frac{2 v}{H} U^{(S)}\left[-4 \gamma^{2} \exp \{-\gamma(c+H)\} \sin \gamma(c+H)\right. \\
& \left.+3 \gamma^{2} \exp \{-2 \gamma(c+H)\}\right],
\end{aligned}
$$

where the Stokes drift (39) and the Stokes flux in the shallow-water approximation become 


$$
\begin{aligned}
& u^{(S)}=\frac{\omega \cosh 2 k(c+H)}{2 k H^{2}} \eta_{0}^{2} \exp (-2 \alpha a-2 \beta t), \\
& U^{(S)}=\frac{\omega}{2 k H} \eta_{0}^{2} \exp (-2 \alpha a-2 \beta t) .
\end{aligned}
$$

The mean drift equation (114) has apparently not been published before. Utilizing the linear wave solutions, we can calculate the dynamic boundary condition at the surface from (15). The contributions from the nonlinear wave terms cancel to lowest order, and we find, assuming negligible vertical mean velocity at the surface, that

$\bar{T}=\rho v W_{c}, \quad c=0$,

where $\bar{T}$ is the complex mean surface wind stress given by $\bar{T} \equiv \bar{\tau}_{1}+i \bar{\tau}_{2}$.

The total mean horizontal displacements are defined as

$Q_{1}=\int_{-H}^{0} \bar{x} d c, \quad Q_{2}=\int_{-H}^{0} \bar{y} d c$

By integrating (114) and assuming zero mean tangential wind stress, i.e. $W_{c}(c=0)=0$ from (117), we then obtain to $O(k / \gamma)$ :

$$
\begin{aligned}
& Q_{1 t t}-f Q_{2 t}=-C_{0}^{2} h_{a}^{(L)}+\frac{1}{2} C_{0} U_{a}^{(S)}+U_{t}^{(S)}+\frac{1}{H} v \gamma U^{(S)}-\tau_{1 B} / \rho, \\
& Q_{2 t t}+f Q_{1 t}=-\tau_{2 B} / \rho .
\end{aligned}
$$

We have here defined the bottom stresses

$\tau_{1 B}=\rho v \bar{x}_{t c}(c=-H), \quad \tau_{2 B}=\rho v \bar{y}_{t c}(c=-H)$.

Utilizing the general relation (44), and noticing that $\tau_{w} / \rho=v \gamma U^{(S)} / H$ in the shallow-water approximation, (119) becomes:

$$
Q_{1 t t}-f Q_{2 t}=-C_{0}^{2} h_{a}^{(L)}-\frac{1}{2} C_{0} U_{a}^{(S)}+\tau_{D} / \rho-\tau_{1 B} / \rho
$$


It is interesting to compare our results with the Eulerian calculations in Phillips (1977). In that case we must insert for the Eulerian mean sea level from (55). We then obtain from (122):

$Q_{1 t t}-f Q_{2 t}=-C_{0}^{2} h_{a}^{(E)}-\frac{3}{2} C_{0} U_{a}^{(S)}+\tau_{D} / \rho-\tau_{1 B} / \rho$

We observe that the second term on the right-hand side of (123) is equal to the divergence of the radiation stress component per unit density $S_{11}=3 E /(2 \rho)=3 C_{0} U^{(S)} / 2$ in shallowwater waves along the 1-axis by Longuet-Higgins and Stewart (1960). If we take $\tau_{D}=\tau_{1 B}=$ 0 , we find for steady, non-rotating motion that

$h_{a}^{(E)}=-S_{11 a} / C_{0}^{2}$

which is Phillips' equation (3.7.5) (here $\partial / \partial a \approx \partial / \partial x$ ). Accordingly, $h^{(E)}=-S_{11} / C_{0}^{2}$, which means that the Eulerian mean water level is depressed in this case (a surface set-down).

\subsection{The non-rotating case}

We do not consider the initial value problem here, but discuss the drift solution for a steady state. The wave amplitude is taken not to vary with time, i.e. $\beta=0$, and we consider the non-rotating case first. Then (105) reduces to

$$
\begin{aligned}
v u_{c c}= & g h_{a}^{(L)}-\frac{1}{2 H} C_{0} U_{a}^{(S)}+v u_{c c}^{(S)} \\
& +\frac{2}{H} v U^{(S)}\left[-4 \gamma^{2} \exp \{-\gamma(c+H)\} \sin \gamma(c+H)\right. \\
& \left.+3 \gamma^{2} \exp \{-2 \gamma(c+H)\}\right] .
\end{aligned}
$$


We note that the second term on the right-hand side is the gradient per unit depth of the radiation stress component per unit density $S_{22}=E /(2 \rho)=C_{0} U^{(S)} / 2$ in shallow-water waves (Longuet-Higgins and Stewart, 1960).

In the case where the form stress from the wind acts to sustain the waves against decay, i.e. $\alpha=\beta=0$, and $\delta=\omega /(2 H \gamma)$ from (34), we regain Longuet-Higgins' (1953) steady drift solution for permanent waves. Disregarding any mean wind stress, the boundary condition are $u(c=-H)=0$, and $u_{c}(c=0)=0$. We then find, correct to $O\left(\varepsilon^{2}\right)$ :

$$
\begin{aligned}
u= & \frac{1}{2 H} U^{(S)}[5-8 \exp \{-\gamma(c+H)\} \cos \gamma(c+H) \\
& +3 \exp \{-2 \gamma(c+H)\}] .
\end{aligned}
$$

As pointed out by Longuet-Higgins, the solution for the drift in permanent waves outside the viscous bottom layer is independent of the value of the viscosity coefficient, as long as it is non-zero.

Without any fluctuating wind $(\delta=0)$, and no temporal decay, the spatial attenuation coefficient in shallow water becomes $\alpha=k /(4 \gamma H)$, as mentioned before. In that case the radiation stress term in (125) becomes one order of magnitude larger than the Stokes drift term. Hence, to obtain a steady state solution in (125), we must assume that the radiation stress term is balanced by the pressure gradient associated with the wave-induced change in mean sea level. This means that a steady-state solution requires

$h^{(L)}=U^{(S)} /\left(2 C_{0}\right)$.

Hence the Lagrangian mean surface position is elevated (a water level set-up).

\subsection{The drift in a rotating ocean}


In a rotating ocean the balance between the Lagrangian mean surface slope and the gradient of the radiation stress $S_{22}$, i.e. (127), still must hold for a steady state solution. For a wave amplitude that does not attenuate in time, (114) yields for the steady drift:

$$
\begin{aligned}
v W_{c c}-i f W= & v u_{c c}^{(S)}+\frac{2}{H} v U^{(S)}\left[-4 \gamma^{2} \exp \{-\gamma(c+H)\} \sin \gamma(c+H)\right. \\
& \left.+3 \gamma^{2} \exp \{-2 \gamma(c+H)\}\right]
\end{aligned}
$$

Introducing the Ekman depth $D=(2 v / f)^{1 / 2}$, applying the no-slip condition $W(c=-H)=$ 0 at the bottom, and assuming vanishing mean tangential wind stress at the surface, i.e., $T=0$ in (117), we readily find from (128):

$$
\begin{aligned}
W= & \frac{1}{H} U^{(S)}[F \exp R c+G \exp (-R c)+N \cosh 2 k(c+H)] \\
& +\frac{1}{2 H} U^{(S)}[-8 \exp \{-\gamma(c+H)\} \cos \gamma(c+H) \\
& +3 \exp \{-2 \gamma(c+H)\}] .
\end{aligned}
$$

Here we have defined the complex coefficients

$R=(1+i) / D, N=2 k^{2} D^{2} /\left(2 k^{2} D^{2}-i\right)$,

$F=G-\frac{2 k N}{R} \sinh k H$

$G=\frac{1}{2 \cosh R H}\left[\frac{2 k N}{R} \sinh k H \exp (-R H)-N+\frac{5}{2}\right]$.

The effect of rotation introduces the Ekman length scale $D$ into the problem, and we realize that the form of the solution now depends crucially on the value of the non-dimensional parameter $H / D$. When $H / D$ is much smaller than unity, the effect of rotation is negligible. On the other hand, when $H / D \gg 1$, the mean drift current will be strongly affected by the earth's rotation. We then have a well-developed Ekman spiral near the bottom, where the 
mean current is deflected somewhat to the right (in the northern hemisphere) of the wave propagation direction, while it practically vanishes in the upper part of the layer. For a wave field where the wave amplitude does not change in space or time, an expression for the mean wave-driven Eulerian current in shallow water has been derived by Xu and Bowen (1994). Their Eulerian current satisfies a no-slip condition at the bottom. In the non-rotating case, the solution by $\mathrm{Xu}$ and Bowen is equal to that given by Phillips (1977), his eqn. (3.4.33). The mean Lagrangian current in Xu and Bowen's (and Phillips') analyses is obtained by adding the inviscid Stokes drift. Hence, the Lagrangian mean drift current does not satisfy a no-slip condition at the bottom, as it physically should. In Fig. 7 we have plotted the Lagrangian mean drift current from (129), non-dimensionalized by $U^{(S)} / H$, as function of the nondimensional depth $c / H$. We have taken $k H=2 \pi / 50$, and $H / D=2.8$. The non-dimensional viscous boundary layer thickness in this case is $1 /(\gamma H)=0.014$.

\section{(Figure 7)}

We notice a well-developed Ekman spiral in the lower half of the water column. It is also worth pointing out that drift in the non-rotating case is quite different in the case when $v \rightarrow 0$ (solid black line) and when $v=0$ (black dashed line).

In the present section we have looked for non-frictional forcing terms for the Lagrangian mean velocity that are depth-varying, and not directly proportional to the Stokes drift. This has been motivated by the results of Mellor (2003, 2005), among others, where he applies a coordinate transformation for the vertical coordinate, and finds vertically-varying radiations stress terms in the Eulerian equations for the mean flow. We find no such terms in the equations for the Lagrangian mean flow. In our approach, the radiation stress term in the equation for the mean drift is depth-independent. As suggested by Weber et al. (2006), we 
find that the radiation stress in shallow water plays a much more important part than in the deep ocean.

\section{The Coriolis-Stokes force in ocean modelling}

Numerical ocean circulation models are generally based on the Eulerian description of motion, and do not resolve the wave-induced drift, which is basically a Lagrangian phenomenon. To compensate for that, the inclusion of the Coriolis-Stokes (CS) force in the equations for the Eulerian mean motion in a rotating ocean has become everyday practice, and goes back for a long time; see e.g. Hasselmann (1970), Huang (1979), Jenkins (1989), McWilliams et al. (1997), McWilliams and Restrepo (1999), Polton et al. (2005), Saetra et al. (2007). Denoting the Eulerian mean velocities by $u_{E}$ and $v_{E}$, and assuming spatially homogeneous flow, one finds to $O\left(\varepsilon^{2}\right)$ for the horizontal Eulerian mean flow (Huang, 1979):

$v \partial^{2} u_{E} / \partial z^{2}-\partial u_{E} / \partial t+f v_{E}=0$

$v \partial^{2} v_{E} / \partial z^{2}-\partial v_{E} / \partial t-f u_{E}=f u^{(S)}$

The term on the right-hand side of (132) is the CS force, and here the Stokes drift in deep water is expressed in the Eulerian coordinates, i.e. $u^{(S)}=\eta_{0}^{2} \omega k \exp 2 k z$, where $z \leq 0$. Directed at right angles to the direction of wave propagation (in the northern hemisphere), the CS force leads to an additional deflection of the mean current, similar to the direct effect of the Coriolis force leading to the Ekman spiral. It should be noted that (131)-(132) has the same structure as the quasi- Eulerian mean current (75) in a Lagrangian description. In the original study (Hasselmann, 1970), the friction terms in (131)-(132) were absent. By keeping the acceleration terms, Hasselmann (1970) showed that the Lagrangian mean flow (Eulerian + Stokes) was purely inertial, i.e. the net drift over the inertial period was zero. By using 
prognostic wave models for the surface wave spectrum like WAM (Komen et al. 1994), or SWAN (Booij et al. 1999), the CS force in (132) can be obtained in spectral form. The overall effect of the CS force on the upper ocean current profiles is substantial, as illustrated by e.g. Polton et al. (2005; their Fig. 3). Estimates from field data collected in northern Norway, with significant wave heights between 1-2 $\mathrm{m}$, demonstrate that the CS force can be of similar magnitude as the ordinary Coriolis force in the Eulerian part of the mean flow (Röhrs et al., 2012).

For pure wave-induced Eulerian mean flow, the appropriate boundary condition at the mean surface level $z=0$, pertaining to (131)-(132), is not at all obvious. By comparing with results from a purely Lagrangian analysis of the drift in permanent waves, it is demonstrated that the relevant boundary condition for wave-driven Eulerian mean flow is that of vanishing shear at the mean surface level (Weber et al. 2015).

\section{Summary and concluding remarks}

In this survey of wave-induced mean drift in a Lagrangian formulation we have stressed the profound difference between surface waves in inviscid fluids, and in fluids with a small viscosity. Although the effect of a weak viscosity only introduces small deviations from linear inviscid, irrotational wave motion (except in cases with surface films), the wave-induced mean motion to second order in wave steepness is substantially affected. In particular, mean momentum is transferred from the classic Stokes drift to Eulerian mean currents via the action of the virtual wave stress (Longuet-Higgins, 1969). The latter depends on the viscosity of the fluid.

Due to the fact that neighboring particles do separate in time, the Lagrangian equations in its primitive form are not well adapted for numerical modelling of large scale ocean flows. 
However, for wave-driven ocean flows to second order; see e.g. (11)-(13), it is possible to compute numerically the nonlinear forcing terms, and then solve the equations for the Lagrangian mean drift velocities numerically in a bounded domain. This constitutes an interesting future research.

\section{Acknowledgements}

Travel support from the Research Council of Norway through the Grant 280625 (Dynamics of floating ice) is gratefully acknowledged. The author is also grateful for the invited visit to ESI at the University of Vienna that initiated this work. The novel results in Secs. 4, 5 and 9 originate from the fruitful and ongoing collaboration with Kai H. Christensen and Göran Broström.

\section{References}

Abrashkin, A. A., 1996. Three-dimensional Gouyon waves. Fluid Dyn. 31, 583-587.

Abrashkin, A.A., Pelinovsky, E., 2017. Lagrange form of the nonlinear Schrödinger equation for low-vorticity waves in deep water. Nonlinear Processes in Geophysics 24, 255 264.

Aiki, H., Greatbatch, R.J., 2012. Thickness-weighted mean theory for the effect of surface gravity waves on mean flows in the upper ocean. J. Phys. Oceanogr. 42, 725-747.

Andrews, D.G., McIntyre, M.E., 1978. An exact theory of nonlinear waves on a Lagrangian-mean flow. J. Fluid Mech. 89, 609-646.

Ardhuin, F., Marié, L., Rascle, N., Forget, P., Roland, A., 2009. Observation and estimation 
of Lagrangian, Stokes, and Eulerian currents induced by wind and waves at the sea surface. J. Phys. Oceanogr. 39, 2820-2838.

Bennet, A., 2006. Lagrangian fluid dynamics. Cambridge University Press, Cambridge, U.K.

Booij, N., Ris, R.C, Holthuijsen, L.H., 1999. A third-generation wave model for coastal regions. 1. Model description and validation. J. Geophys. Res. 104, 7649-7666.

Broström, G., Christensen, K.H., Weber, J.E., 2008. A quasi-Eulerian, quasi-Lagrangian view of surface-wave-induced flow in the ocean. J. Phys. Oceanogr. 38, 1122-1130.

Bye, J.A.T., 1988. The coupling of wave drift and wind velocity profiles. J. Mar. Res. 46, 457-472.

Chalikov, D.V., Makin, V.K., 1990. Numerical approximation of wind-wave interaction parameter. Ann. Geophys. Special issue, 197-198.

Chang, M.-S., 1969. Mass transport in deep-water long-crested random gravity waves. J. Geophys. Res. 74, 1515-1536.

Chen, Y.-Y, Hsu, H.-C., Chen, G.-Y., 2010. Lagrangian experiment and solution for irrotational finite-amplitude progressive gravity waves at uniform depth. Fluid Dyn. Res. 42, doi:10.1088/0169-5983/42/4/045511

Christensen, K.H., 2005. Transient and steady drift currents in waves damped by surfactants. Phys. Fluids 17, 042102.

Christensen, K.H., Weber, J.E., 2005a. Drift of an inextensible sheet caused by surface waves. Environ. Fluid Mech. 5, 495-505.

Christensen, K.H., Weber, J.E., 2005b. Wave-induced drift of large floating sheets. Geophys. Astrophys. Fluid Dyn. 99, 433-443. 
Clamond, D., 2007. On the Lagrangian description of steady surface gravity waves. J. Fluid Mech. 589, 433-454.

Constantin, A., 2001. Edge waves along a sloping beach, J. Phys. A Math. Gen. 34, 97239731.

Constantin, A., 2012. An exact solution for equatorially trapped waves, J. Geophys. Res. Oceans 117, C05029, doi:10.1029/2012JC007879.

Constantin, A. 2014. Some nonlinear, equatorial trapped, nonhydrostatic internal geophysical waves, J. Phys. Oceanogr. 44, 781-789.

Constantin, A., 2015. The flow beneath a periodic travelling surface water wave. J. Phys. A: Math. Theor. 48, doi:10.1088/1751-8113/48/14/143001.

Constantin, A., Monismith, S.G., 2017. Gerstner waves in the presence of mean currents and rotation. J. Fluid Mech. 820, 511-528.

Craik, A.D.D., 1982. The drift in water waves. J. Fluid Mech. 116, 187-205.

Craik, A.D.D, Leibovich, S., 1976. A rational model for Langmuir circulations. J. Fluid Mech. 73, 401-426.

De Carolis, G., Olla, P., Piagnoli, L., 2005. Effective viscosity of grease ice in linearized gravity waves. J. Fluid Mech. 535, 369-381.

Dorrestein, R., 1951. General linearized theory of the effect of surface films on water ripples. Proc. K. Ned. Akad. Wet. Ser. B 54, 260, 350.

Dysthe, K., Rabin, Y., 1986. ONRL Workshop Proceedings-Role of surfactant films on the interfacial properties of the sea surface, eds. Herr, F.L., Williams, J. U.S Office of Naval Res. London 187-213.

Ekman, V.W., 1905. On the influence of the earth's rotation on ocean-currents. Ark. Mat. 
Astron. Fys. 2, 1-53.

Euler, L., 1757. Principes généraux du mouvement des fluides. Mémoires de l'Académie des Sciences de Berlin 11, 274-315.

Faller, A.J., Caponi, E.A., 1978. Laboratory studies of wind-driven Langmuir circulations. J. Geophys. Res. 83, 3617-3633.

Førland, E., 1989. Wave-induced currents in the ocean. Dr. Scient thesis, Department of Geophysics, University of Oslo, Norway, $182 \mathrm{pp}$.

Gade, M., Hühnerfuss, H., Korenowski, G., Eds., 2006. Marine Surface Films: Chemical Characteristics, Influence on Air-Sea Interactions and Remote Sensing, Springer, Berlin, Heidelberg, New York.

Gaster, M., 1962. A note on the relation between temporally-increasing and spatiallyincreasing disturbances in hydrodynamic stability. J. Fluid Mech. 14, 222-224.

Gerkema, T., Zimmerman, J.T.F, Maas, L.R.M., van Haren, H., 2008. Geophysical and astrophysical fluid dynamics beyond the traditional approximation. Rev. Geophys. 46, RG2004-33.

Gerstner, F., 1809. Theorie der Wellen samt einer daraus abgeleiteten Theorie der Deichprofile, Ann. Phys. 2, 412-455.

Hasselmann, K., 1970. Wave-driven inertial oscillations. Geophys. Fluid Dyn. 1, 463-502.

Huang, N.E., 1979. On surface drift currents in the ocean. J. Fluid Mech. 91, 191-208.

Henry, D., 2013. An exact solution for equatorial geophysical water waves with an underlying current, Eur. J. Mech. B Fluids 38, 18-21.

Henry, D., 2016. Equatorially trapped nonlinear water waves in a $\beta$-plane approximation with centripetal forces, J. Fluid Mech. 804, R1, 11 pp. 
Høydalsvik, F., Weber, J. E., 2003. Mass transport velocity in free barotropic Poincaré waves. J. Phys. Oceanogr. 33, 2000-2012.

Huang, N.E., 1979. On surface drift currents in the ocean, J. Fluid Mech. 91, 191-208.

Ionescu-Kruse, D., 2015. An exact solution for geophysical edge waves in the $\beta$-plane approximation, J. Math. Fluid Mech. 17, 699-706.

Jacobs, S.J., 1987. An asymptotic theory for the turbulent flow over a progressive water wave. J. Fluid Mech. 174, 69-80.

Jenkins, A.D., 1986. A theory for steady and variable wind- and wave-induced currents. J. Phys. Oceanogr. 16, 1370-1377.

Jenkins, A.D., 1987. Wind and wave induced currents in a rotating sea with depth-varying eddy viscosity. J. Phys. Oceanogr. 17, 938-951.

Jenkins, A.D., 1989. The use of a wave prediction model for driving a near-surface current model. Dt. hydrogr. Z. 42, 133-149.

Jenkins, A.D, Jacobs, S.J., 1997.Wave damping by a thin layer of viscous fluid. Phys. Fluids $9,1256-1264$.

Jenkins, A.D., Dysthe, K.B, 1977. The effective film viscosity coefficients of a thin floating fluid layer. J. Fluid Mech. 344, 335-337.

Knight, D., 1977. Turbulent flow over a wavy boundary. Bound.-Layer Meteor.11, 205-222. Komen, G.J., Cavaleri, L., Doneland, M., Hasselmann, K, Hasselman, S., Janssen, P.E.A.M., Eds., 1994. Dynamics and Modelling of Ocean Waves. Cambridge University Press, $533 \mathrm{pp}$.

Kravtchenko, J., Daubert, A., 1957. Closed trajectory waves in a finite depth. La Houille Blanche 3, 408-429.

Lamb, H., 1932. Hydrodynamics, $6^{\text {th }}$ ed. Cambridge University Press, Cambridge, U.K. 
Law, A.W.K., 1999. Wave-induced surface drift of an inextensible thin film. Ocean Engng. $26,1145-1168$.

LeBlond, P.H., Mysak, L.A., 1978. Waves in the ocean. Elsevier, Amsterdam, Holland.

Longuet-Higgins, M.S., 1953. Mass transport in water waves. Phil. Trans. Roy. Soc. Lond. A $245,535-581$

Longuet-Higgins, M.S., 1969. A nonlinear mechanism for the generation of sea waves. Proc. Roy. Soc. A 311, 371-389.

Longuet-Higgins, M.S.,1986. Eulerian and Lagrangian aspects of surface waves. J. Fluid Mech. 173, 683-707.

Longuet-Higgins, M.S., 1988. Lagrangian moments and mass transport in Stokes waves. Part

2. Water of finite depth. J. Fluid Mech. 186, 321-336.

Longuet-Higgins, M.S., Stewart, R.W., 1960. Changes in the form of short gravity waves on long waves and tidal currents. J. Fluid Mech. 8, 565-583.

Lucassen, J., 1968. Longitudinal capillary waves. Part 1-Theory. Trans. Faraday Soc. 64, 2221-2229.

Madsen, O.S., 1977. A realistic model of the wind-induced Ekman boundary layer. J. Phys. Oceanogr. 7, 248-255.

Madsen, O.S., 1978. Mass transport in deep-water waves. J. Phys. Oceanogr. 8, 1009-1015.

McIntyre, M.E., 1988. A note on the divergence effect and the Lagrangian-mean surface elevation in periodic water waves. J. Fluid Mech. 189, 235-242.

McWilliams, J.C., Sullivan, P., Moeng, C.-H., 1997. Langmuir turbulence in the ocean. J. Fluid Mech. 334, 1-30.

McWilliams, J.C., Restrepo, J.M., 1999. The wave-driven ocean circulation. J. Phys. Oceanogr. 29, 2523-2540.

Miche, M., 1944. Mouvement ondulatoires de la mer en profondeur constante ou décroissante. 
Ann. Ponts Chaussées 114, 25-78.

Mellor, G., 2003. The three-dimensional current and surface wave equations. J. Phys. Oceanogr. 33, 1978-1989.

Mellor, G., 2005. Some consequences of the three-dimensional current and surface wave equations. J. Phys. Oceanogr. 35, 2291-2298.

Melsom, A., 1992. Wave-induced roll motion beneath an ice cover. J. Phys. Oceanogr. 22, 19-

28.

Melsom, A., 1993. Effects of variable eddy viscosity on wave-induced currents under ice. Ann. Geophys. 11, 78-88.

Melsom, A., 1996. Effects of wave breaking on the surface drift. J. Geophys. Res. 101, $12,071-12,078$

Melsom, A., Saetra, Ø., 2004. Effects of wave breaking on the near-surface profiles of velocity and turbulent kinetic energy. J. Phys. Oceanogr. 34, 490-504.

Miles, J., 1957. On the generation of surface waves by shear flows. J. Fluid Mech. 3, 185-204.

Ng, C.-O., 2004a. Mass transport and set-ups due to partial standing surface waves in a twolayer viscous system. J. Fluid Mech. 520, 297-325.

Ng, C.-O., 2004b. Mass transport in gravity waves revisited. J. Geophys. Res. 109, C04012, doi:10.1029/2003JC002121.

Ng, C.-O., Zhang, X., 2007. Mass transport in water waves over a thin layer of soft viscoelastic mud. J. Fluid Mech. 573, 105-130.

Phillips, O.M., 1957. On the generation of waves by turbulent wind. J. Fluid Mech. 2, $417-$ 445.

Phillips, O.M., 1977. The dynamics of the upper ocean, $2^{\text {nd }}$ ed. Cambridge University Press, Cambridge, U.K. 
Piedra-Cueva, I., 1995. Drift velocity of spatially decaying waves in a two-layer viscous system. J. Fluid Mech. 299, 217-239.

Pierson, W.J., 1962. Perturbation analysis of the Navier-Stokes equations in Lagrangian form with selected solutions. J. Geophys. Res. 67, 3151-3160.

Pollard, R. T., 1970. Surface waves with rotation: an exact solution. J. Geophys. Res. 75, $5895-5898$.

Polton, J.A.D., Lewis, M., Belcher, S.E., 2005. The role of wave-induced Coriolis-Stokes forcing on the wind-driven mixed layer. J. Phys. Oceanogr. 35, 444-457.

Röhrs, J., Christensen, K.H, Hole, L.R., Broström, G., Drivdal, M., Sundby, S., 2012. Observation-based evaluation of surface wave effects on currents and trajectory forecasts. Ocean Dyn. 62, 1519-1533.

Saetra, Ø., Albretsen, J., Janssen, P.A.E.M., 2007. Sea-state dependent momentum fluxes for ocean modeling. J. Phys. Oceanogr. 37, 2714-2725.

Sebille, E. van, Griffies, S.M., Abernathey, R., Adams, T.P., Berloff, P., Biastoch, A., Blanke, B., Chassignet, E.P., Cheng, Y., Cotter, C.J., Deleersnijder, E., Döös, K., Drake, H.F., Drijfhout, S., Gary, S.F., Heemink, A.W., Kjellsson. J., Koszalka, I.M., Lange, M., Lique, C., MacGilchrist, G.A., Marsh, R., Adame, C.G.M., McAdam, R., Nencioli, F., Paris, C.B., Piggott, M.D., Polton, J.A., Rühs, S., Shah, S.H.A.M., Thomas, M.D., Wang, J., Wolfram, P.J., Zanna, L., Zika, J.D., 2018. Lagrangian ocean analysis: Fundamentals and practices. Ocean Modelling 121, 49-75.

Starr,V.P., 1959. Hydrodynamical analogy to $E=m c^{2}$. Tellus $11,135-138$.

Stokes, G.G., 1847. On the theory of oscillatory waves. Trans. Cam. Phil. Soc. 8, 441-455.

Ünlüata, Ü., Mei, C.C., 1970. Mass transport in water waves. J. Geophys. Res. 36, 7611-7618. Ursell, F., 1950. On the theoretical form of ocean swell on a rotating earth. Mon. Not. R. 
Astron. Soc., Geophys. Suppl. 6, 1-8.

Weber, J.E., 1983a. Attenuated wave-induced drift in a viscous rotating ocean. J. FluidMech. $137,115-129$.

Weber, J.E., 1983b. Steady wind- and wave-induced currents in the open ocean. J. Phys.

Oceanogr. 13, 524-530.

Weber, J.E., 1985. Friction-induced roll motion in short-crested surface gravity waves. J.

Phys. Oceanogr. 15, 936-942.

Weber, J.E., 1987. Wave attenuation and wave drift in the marginal ice zone. J. Phys. Oceanogr. 17, 2351-2361.

Weber, J.E., 1998. Mass transport induced by surface waves in a viscous rotating fluid. In:

Free Surface Flows with Viscosity, 37-67. Ed. P.A. Tyvand, Computational Mechanics Publications, Southampton, UK.

Weber, J.E., 2001. Virtual wave stress and mean drift in spatially damped surface waves. J. Geophys. Res. 106, 11,653-11,657.

Weber, J.E., 2012. A note on trapped Gerstner waves, J. Geophys. Res. 117, C03048, doi: 10.1029/2011JC007776.

Weber, J.E., Førland, E., 1989. Effect of an insoluble surface film on the drift velocity of capillary-gravity waves. J. Phys. Oceanogr. 19, 952-961.

Weber, J.E., Førland, E. 1990. Effect of air on the drift velocity of water waves. J. Fluid Mech. 218, 619-640.

Weber, J.E., Melsom, A., 1993. Transient ocean currents induced by wind and growing waves. J. Phys. Oceanogr. 23, 193-206.

Weber, J.E., Saetra, Ø., 1995. Effect of film elasticity on the drift velocity of capillary gravity 
waves. Phys. Fluids 7, 307-314.

Weber, J.E., Christensen, K.H., 2003. Mean drift induced by free and forced dilational waves. Phys. Fluids 15, 3703-3709.

Weber, J.E., Broström, G., Saetra, Ø., 2006. Eulerian vs Lagrangian approaches to the waveinduced transport in the upper ocean. J. Phys. Oceanogr. 36, 2106-2118.

Weber, J.E., Christensen, K.H., Broström, G., 2008. Radiation stress and depth-dependent drift in surface waves with dissipation. Int. J. Offshore and Polar Engn. 18, 8-13.

Weber, J.E., Drivdal, M., Christensen, K.H, Broström, G., 2015. Some aspects of the Coriolis-Stokes forcing in oceanic momentum and energy budgets. J. Geophys. Res. Oceans, 120, doi:10.1002/2015JC010717.

Xu, Z., Bowen, A.J., 1994. Wave-and wind-driven flow in water of finite depth. J. Phys. Oceanogr. 24, 1850-1866. 
Figures

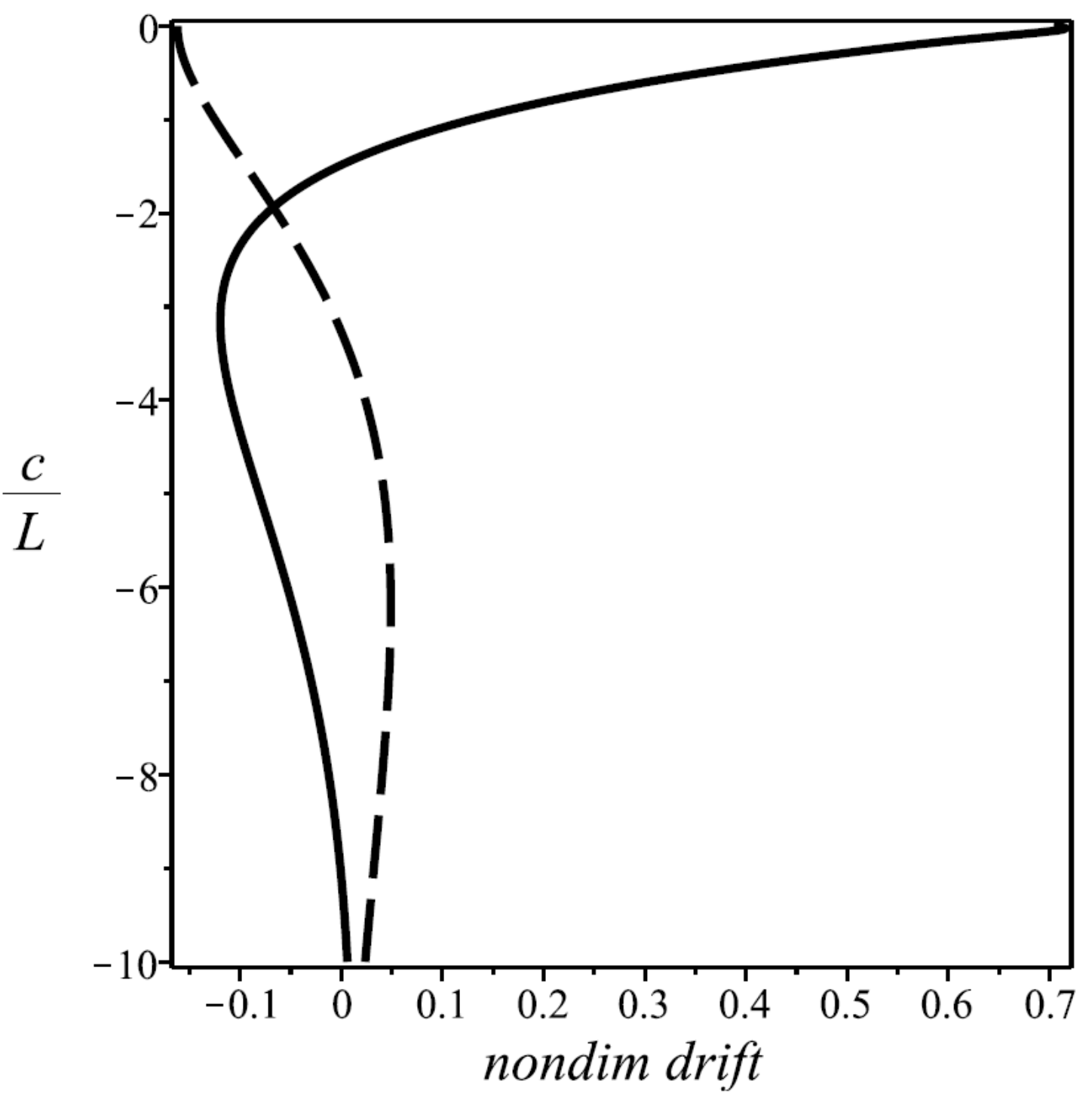

Fig. 1. 


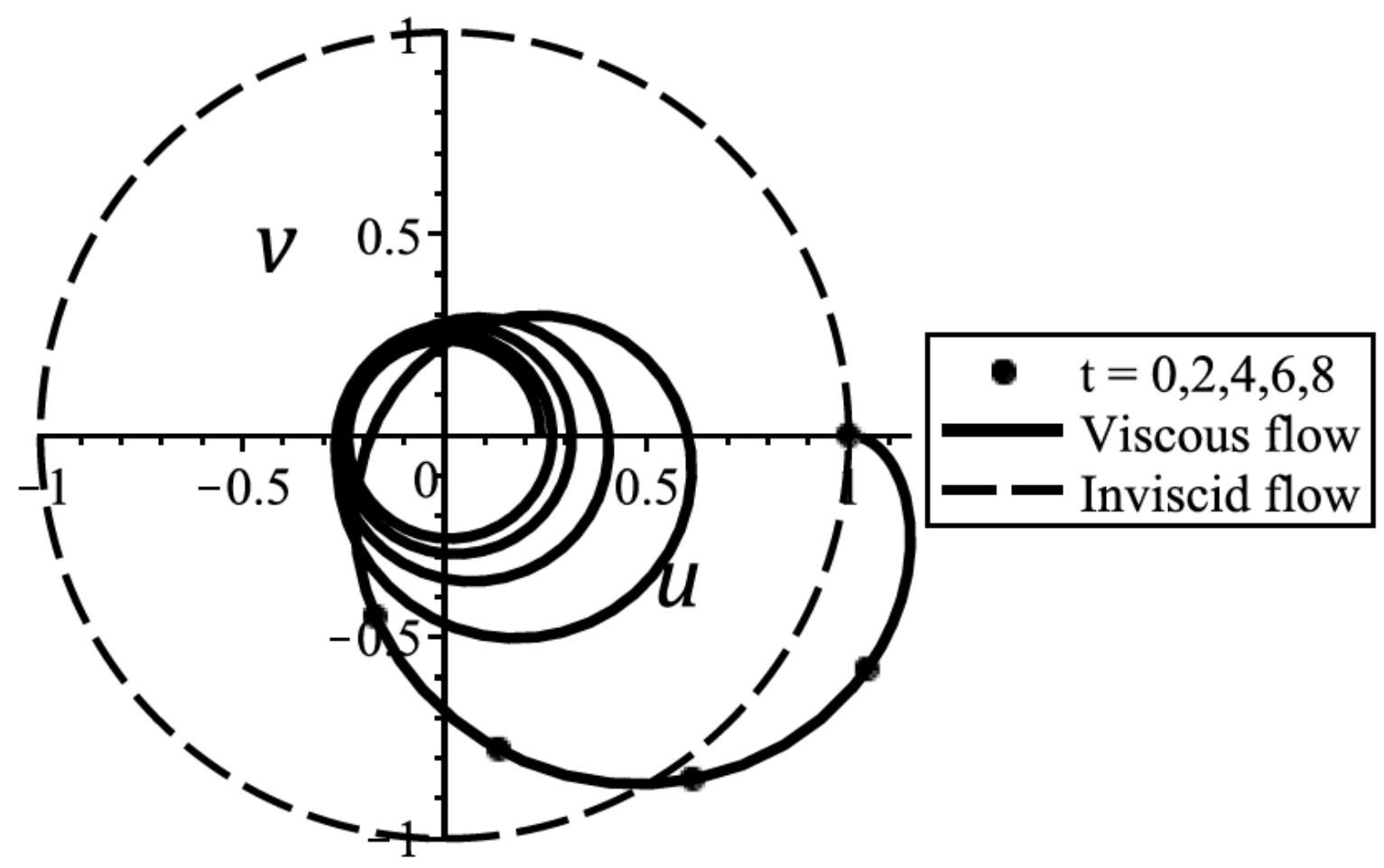

Fig. 2. 


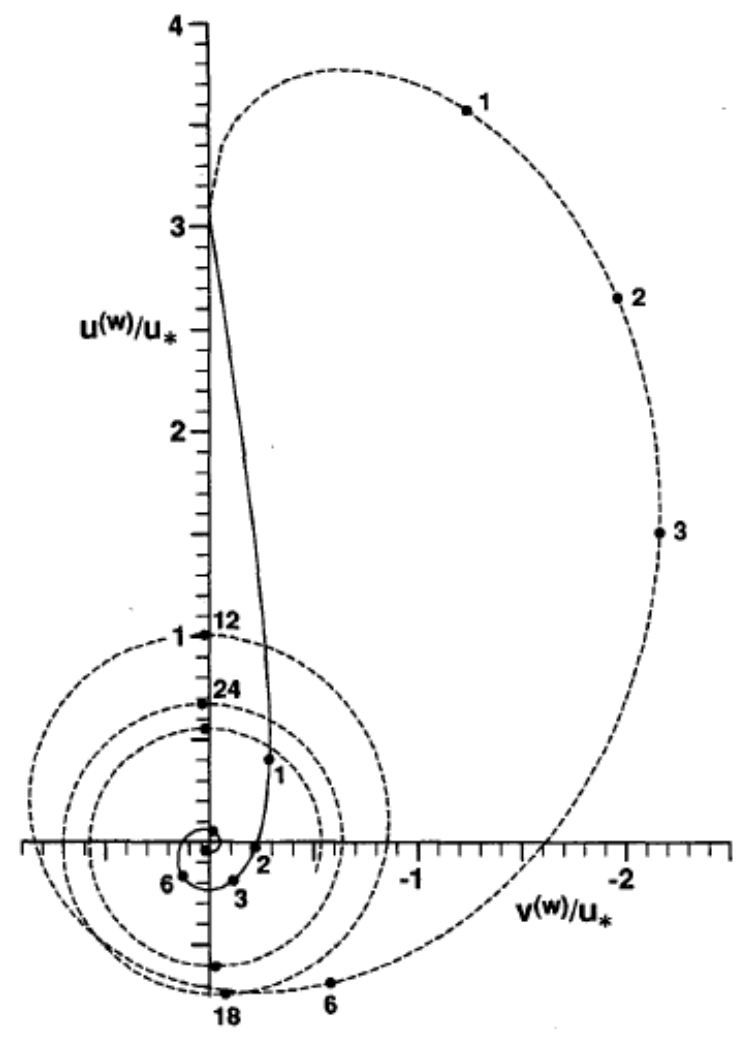

Fig. 3. 


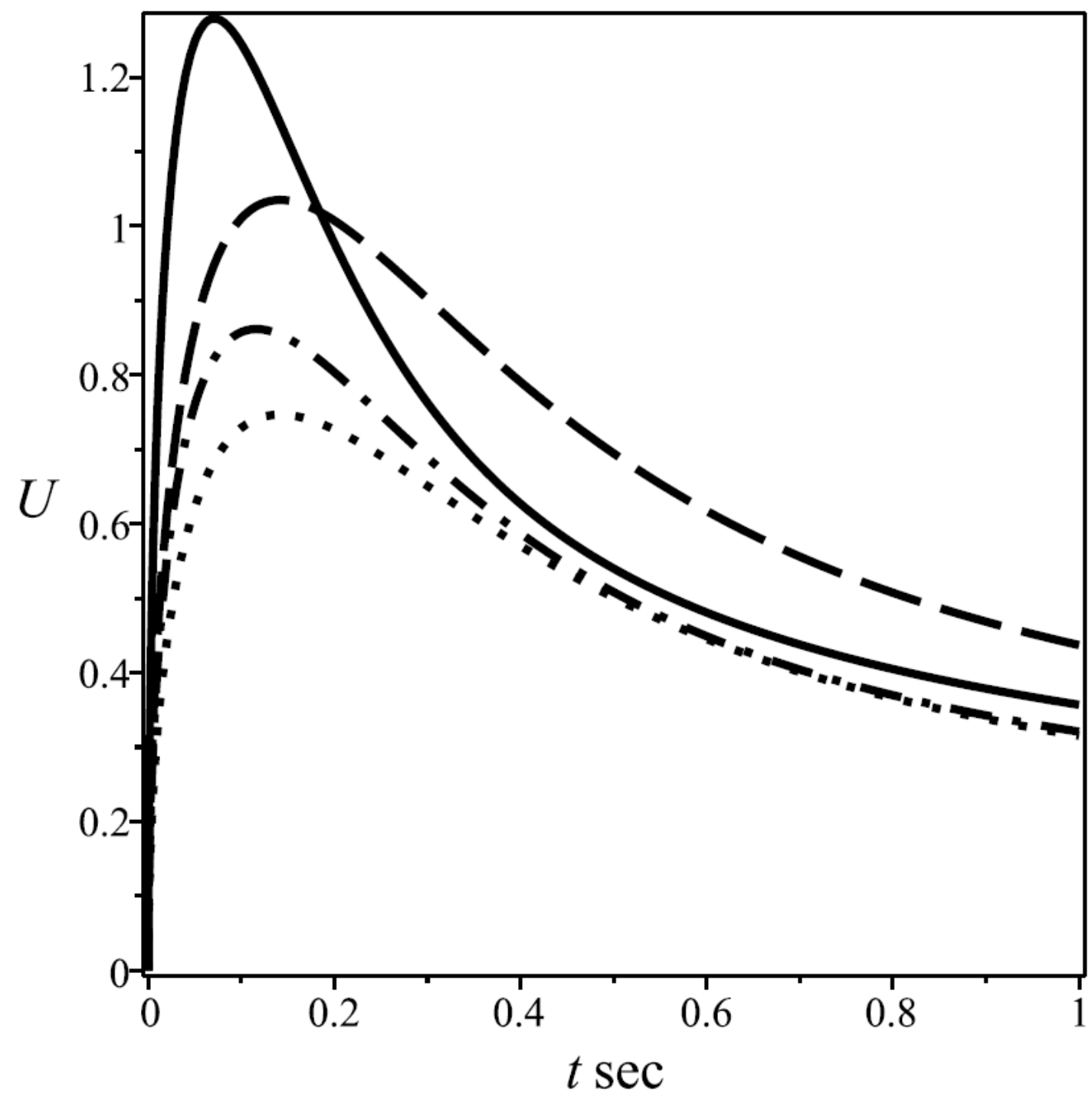

Fig. 4. 


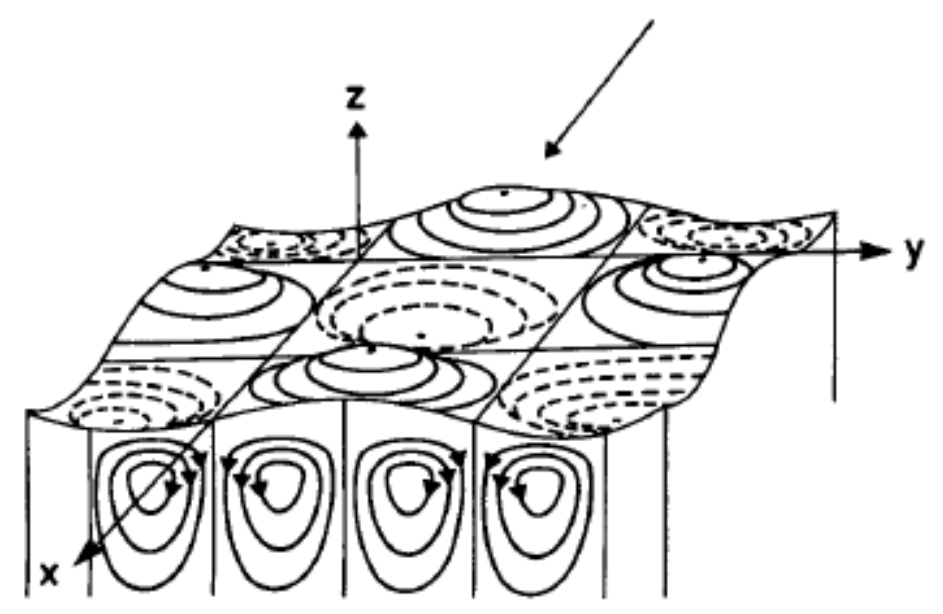

Fig. 5.

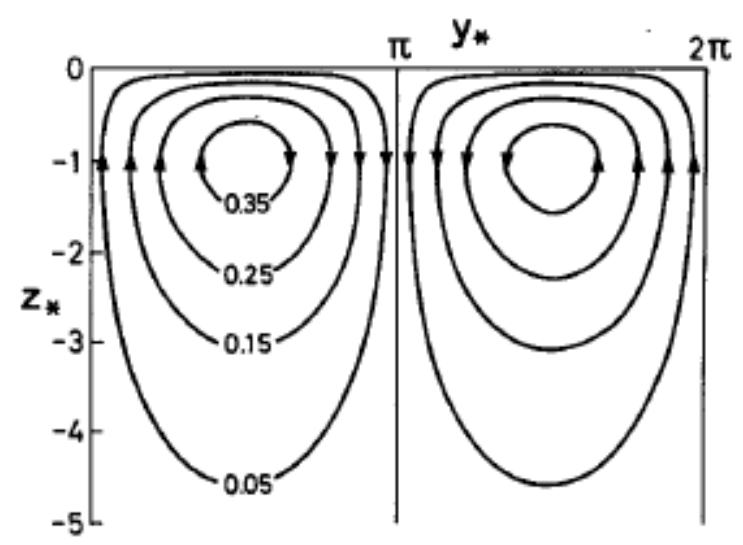

Fig. 6. 


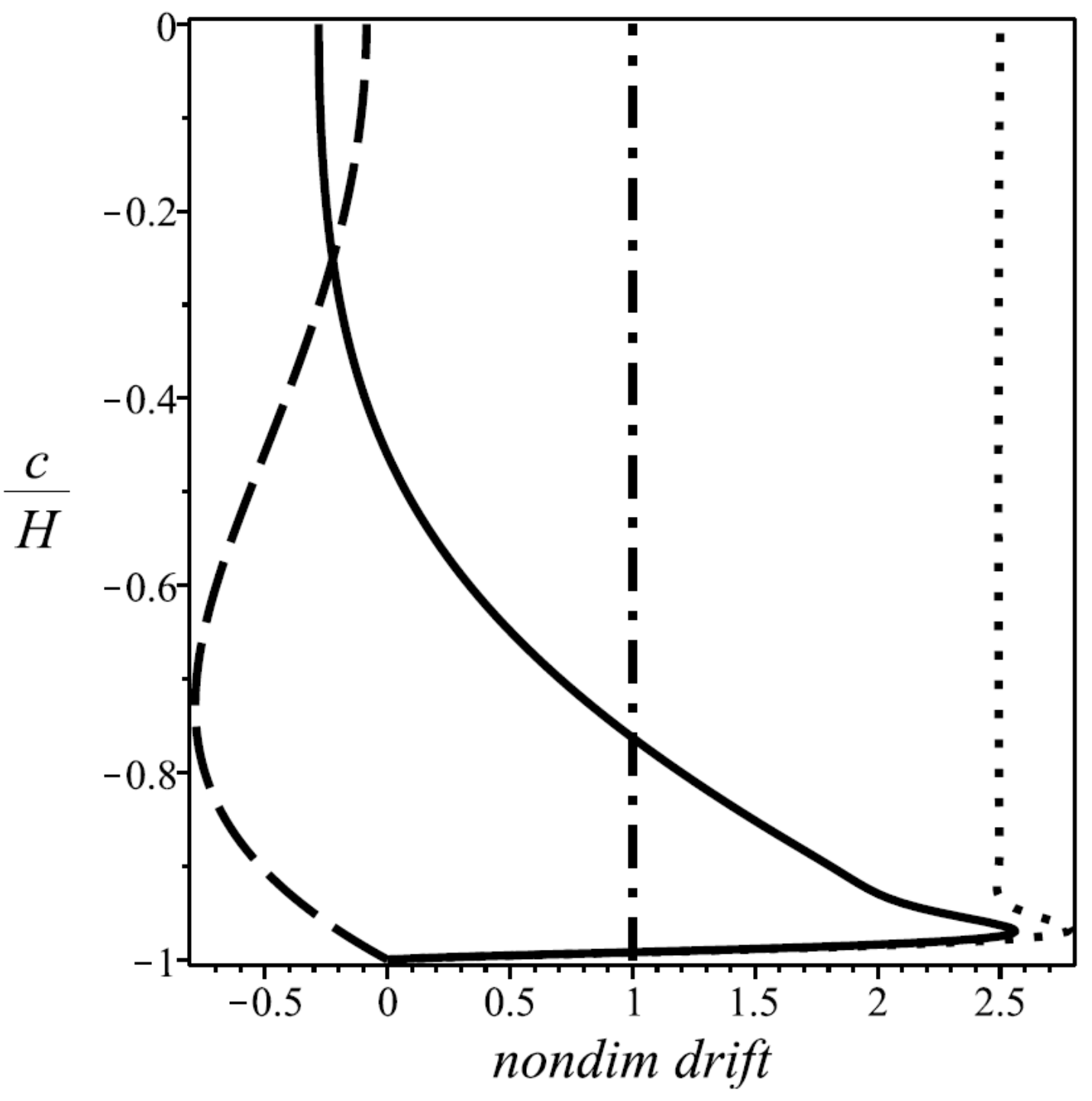

Fig. 7. 


\section{Figure captions}

Fig. 1. Dimensionless wave drift components $u^{(w)} / u_{0}$ (solid line) and $v^{(w)} / u_{0}$ (dashed line) as function of dimensionless depth $c / L$.

Fig. 2. Hodographs of the non-dimensional Lagrangian surface drift current when $v=$ $10^{-3} \mathrm{~m}^{2} \mathrm{~s}^{-1}$ (solid line) and $v=0$ (dashed circle). Black dots on the graph denote time in hours after the onset of mean motion.

Fig. 3. Hodograph of the dimensionless wave-induced surface current $W^{(w)} / u_{*}$ for temporally damped waves caused by adverse winds (solid line). The dashed line represents the equivalent surface current when the eddy viscosity is constant (see also Fig. 2). Numbered black dots denote the time in pendulum hours after the onset of motion (after Weber and Melsom (1993), (CAmerican Meteorological Society, used with permission).

Fig. 4. Non-dimensional quasi-Eulerian surface drift $U$ as function of time for $A_{*}=1$ (solid line), $A_{*}=0.5$ (dashed line), $A_{*}=5$ (dash-dotted line), $A_{*} \rightarrow \infty$ (dotted line). The last value corresponds to the inextensible film case.

Fig. 5. Sketch of induced roll motion with the primary wave field (advancing in the direction of the arrow) (after Weber (1985), (CAmerican Meteorological Society, used with permission).

Fig. 6. Streamlines for dimensionless stream function $\psi_{*}=\psi \gamma /\left(\eta_{0}^{2} \omega K\right)$; see text for details (after Weber (1985), @American Meteorological Society, used with permission). 
Fig. 7. Non-dimensional drift velocity $u$ (solid line) and $v$ (dashed line) vs. non-dimensional depth $c / H$ when $D / H=0.36$, and $k H=2 \pi / 50$. The dotted line is the non-rotating result (126), and the dash-dotted line depicts the inviscid Stokes drift. 